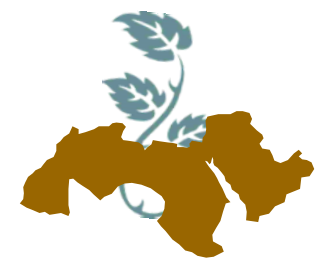

Arab Univ.

J. Agric. Sci.,

Ain Shams Univ., Cairo

Special Issue, 26(2A), 935 - 950, 2018

\title{
EFFECT OF SALINE WATER IRRIGATION AND FOLIAR SPRAYING OF SALICYLIC ACID ON GROWTH, FLOWERING AND CHEMICAL COMPOSITION OF POT MARIGOLD (Calendula officinalis L.) PLANT
}

\author{
Zeinab A. Abou El-Ftouh; Asmaa M. Mohamed \\ and A.K. Ibrahim \\ Horticulture Dept., Fac. of Agric., Ain Shams Univ., Cairo, Egypt
}

Keywords: Pot marigold, Salicylic acid, Salinity, Sodium chloride, Calcium chloride, Proline content

\section{ABSTRACT}

This study was carried out to investigate the effect of saline water irrigation at 2000, 4000, 6000,8000 and 10000 ppm by using a mixture of two salts (sodium chloride: calcium chloride, 2:1, $\mathrm{w} / \mathrm{w}$ ) in addition to tap water as control and foliar sprayings of Salicylic acid (SA) at 100, 200 and $300 \mathrm{ppm}$ in addition to tap water as control on growth, flowering and chemical composition of pot marigold plants in the two successive seasons of 2014/2015 and 2015/2016 in the Faculty of Agriculture, Ain Shams University, Qalyubia Governorate, Egypt. Results revealed that all tested foliar applications of SA increased all vegetative growth characteristics (plant height, number of leaves /plant, leaf area, number of branches/plant, diameter of stem, fresh and dry weights of vegetative growth and roots, and roots volume), and flowering attributes (number of inflorescences/plant, diameter of inflorescence, fresh and dry weights of inflorescences). While, saline water irrigation decreased growth, flowering parameters, chlorophyll, carotene contents in flowers and some minerals contents of the vegetative growth, i.e. nitrogen, phosphorus and potassium percentages. However, saline water irrigation increased proline and some mineral contents, i.e. calcium, sodium and chloride percentages of the vegetative growth. In most cases, under the same saline water irrigation conditions spraying SA at 100 and 200 ppm concentrations alleviated the salinity effect on the plants, enhanced the plant growth and increased the flowering parameters. The most effective treatments which enhance growth, flowering parameters, chlorophyll content, carotene contents in flowers, proline and mineral contents were found to be 100 and 200 ppm SA spraying under nonsaline conditions.

\section{INTRODUCTION}

Pot marigold (Calendula officinalis L.) belongs to Asteraceae (Compositae) family and is considered one of the most popular winter annual flowering plants, it is grown as an ornamental plant in beds and boarders. The pot marigold is natively from the Mediterranean region and grows widely across Europe and North America as an ornamental and medicinal plant (Earle et al 1964). It grows in sunny or partial shade locations and is easy to grow requiring little cultivation practices (Dole and Wilkins, 2004). The plants produce colored flowers from bright yellow to orange, which are used as attractive cut flowers. Also, it is an important medicinal and ornamental plant (Kishimoto et al 2005).

Salinity is an important environmental factor which limits plant growth and productivity (Allakhverdiev et al 2000 and Kaya et al 2003). Saline soil is the soil which have an electrical conductivity of the saturated paste extract (EC) of $4 \mathrm{dSm}^{-1}$ (4 $\mathrm{dSm}^{-1} \sim 40 \mathrm{mM} \mathrm{NaCl}$ ) or more. In most arid and semiarid areas, there is a competition between agriculture and industry for use of high quality water and that accentuated the salinity problem. The use of alternative water sources for irrigation has been promoted by landscape users. Thence, marginal quality water, somewhat saline water, will become important in arid and semiarid areas (Chartzoulakis et al 2002) and can be used for 
the irrigation of ornamental plants (Carter et al 2005). However, the use of low quality water for irrigation affects plants in different ways, depending on the degree of salt tolerance of the species (Alarcon et al 1994) and even within a given species (Sanchez-Blanco et al 2003). Salt stress can negatively affect plant survival, biomass, plant height, plant morphology, photosynthesis, protein synthesis, energy and lipid metabolism, and the plant's ability to absorb water and nutrients (Parida and Das, 2005).

Salicylic acid (SA) is a phenolic derivative and it is distributed in the whole plant kingdom, also it is classified under the group of plant hormones. Salicylic acid has been found to play a key role in the regulation of plant growth, development and in the responses to environmental stresses (RivasSanVicente and Plasencia, 2011 and Hara et al 2012). The influence of $S A$ application on plants differ according to the concentration of applied SA and the method of application, such as foliar spray and hydroponic culture. These methods depend on the plant species; therefore, conflicting results can be reported. In general low concentrations of SA enhances plant tolerance to abiotic stresses, while high concentrations of SA stimulate inhibitory effects on plant growth and reduce tolerance of SA (Khan et al 2010 and Nazar et al 2011). SA may impact a range of diverse processes in plants; stomatal closure, ion uptake and transport, membrane permeability, photosynthetic and growth rates. Exogenous application of salicylic acid improved growth and yield of various ornamental plants and vegetable crops.

\section{MATERIALS AND METHODS}

Pot experiments were carried out during the two successive seasons of 2014/2015 and $2015 / 2016$. Trials took place in the ornamental farm of the Horticulture Department, Faculty of Agriculture, Ain Shams University, Shoubra Elkheima, Qalyubia Governorate, Egypt. Local seeds of Pot marigold (Calendula officinalis L.) were sown in the nursery on October $1^{\mathrm{St}}$ in both growing seasons. The seedlings were transplanted in the pots after 45 days from sowing, the seedlings were planted individually in black plastic pots $20 \mathrm{~cm}$ diameter filled with sand medium.

Treatments were arranged in a completely randomized block design with three replicates, each replicate represented by 120 pots. After 120 days from planting, vegetative and root growth parame- ters were recorded included plant height, number of leaves per plant, leaf area, number of branches per plant, stem diameter, fresh and dry weight of vegetative and roots growth and roots volume. When inflorescences were full opening, number of inflorescence/plant, diameter of inflorescence, and fresh and dry weights of inflorescence were determined. Minerals content were determined represented in nitrogen, phosphorus, potassium, calcium, sodium and chloride percentages in the vegetative growth. Average leaf area $\left(\mathrm{cm}^{2}\right)$ was estimated using the disk method according to Moursi et al (1968). Total chlorophyll content was measured in leaves and carotene contents were measured in flowers. Total nitrogen was determined using Kjeldahl method as described by piper (1950). Phosphorus content was measured spectrophotometrically using the ascorbic acid method (AOAC, 2005). Potassium was measured by flame photometer as described by Page et al (1982). Sodium was measured by flame photometer as described by Brown and Lilleland (1946). Chloride was measured by Mohr's method as described by Kraemer and Stamm (1924). Calcium was measured by the Versenate (EDTA) method as described by Cheng and Bray (1951). Leaf chlorophyll content was determined in the $5^{\text {th }}$ fresh leaf (mg/g fresh weight) according to the method described by Wintermans and De Mots (1965). Carotene contents in flowers were determined as mentioned by Nagata and Yamashita (1992). Proline was estimated colorimetrically in fresh samples as described by Batels et al (1973).

Six concentrations of saline water irrigation were used tap water as control, 2000, 4000, 6000, 8000 and $10000 \mathrm{ppm}$. The saline water irrigation were prepared by using a mixture of two salts (sodium chloride: calcium chloride, $2: 1, w / w)$ as described by Kandeel (2004).

Four concentrations of foliar sprayings of salicylic acid were used 0, 100, 200 and 300 ppm. Salicylic acid was dissolved in absolute ethanol and then added to water (ethanol: water, 1: 1000, $\mathrm{v} / \mathrm{v}$ ) as described by Williams et al (2003). Spraying of SA was done after two weeks from transplanting and was repeated every two weeks. Foliar sprayings were applied during early morning hours using a hand-held sprayer. In order to avoid interferences with different moisture levels, the same amount of distilled water was sprayed to the control plants at a given time. The spray solution was maintained just to cover completely the plant foliage. 
Effect of Saline Water Irrigation and Foliar Spraying of Salicylic Acid on Growth,

The statistical analysis was conducted using the CoStat package program (Version 6.303; CoHort Software, USA). Data were subjected to analysis of variance (ANOVA). The differences among means of data were compared by Duncan's Multiple Range Test (Waller and Duncan, 1969). All statistical determinations were made at $\mathrm{P}=0.05$.

\section{RESULTS AND DISCUSSION}

\section{Vegetative growth}

Data in Tables (1 and 2) show that irrigation by saline water at all concentrations levels significantly decreased all vegetative characters compared with the irrigation with tap water treatment in both seasons. While all tested foliar spraying of salicylic acid treatments significantly increased all vegetative characters compared with the control treatment in both seasons. Foliar spraying of SA at 100 ppm gave the highest values of plant height, number of leaves/plant, leaf area, number of branches/plant, stem diameter, fresh and dry weights of the vegetative growth. In most cases, under the same saline water irrigation concentration, spraying SA alleviated the effect of saline water irrigation on the plants and enhance the plant growth as compared with control treatment in both seasons. The SA spraying treatments which gave the highest alleviation of the effect of saline water irrigation on plants were 100 and 200 ppm. Furthermore, the interactive effect of saline water irrigation and foliar spraying of SA which gave the highest significant value of plant height, number of leaves/plant, leaf area, number of branches/plant, stem diameter, fresh and dry weights of the vegetative growth was obtained from the application of spraying SA 100 $\mathrm{ppm}$ with tap water irrigation treatment in both seasons.

As for the saline water irrigation effect on the vegetative growth characteristics, the obtained results are in agreement with those reported by Bayat et al (2012), Hashish et al (2015b), and Nofal et al (2015) for pot marigold. Also, similar results were reported by Najafian et al (2009a) for rosemary, Najafian et al (2009b) for thyme, Fatemi and Aboutalebi (2012) for sweet basil, Salachna et al (2015) for speckled spur-flower. Concerning the effect of foliar spraying of SA on the vegetative growth characteristics, the obtained results are in agreement with those reported by Bayat et al (2012), Hashish et al (2015b), and Nofal et al (2015). In addition, similar findings were reported by Fathy et al (2003) for broad bean, Szepesi et al (2005), He and Zhu (2008) and Mady (2009) for tomato, Yildirim et al (2008) for cucumber, Elwan and El-Hamahmy (2009) for pepper, Karlidag et al (2009) for strawberry.

Data in Table (3) clearly show that all tested saline water irrigation treatments significantly decreased volume of roots, fresh and dry weights of roots compared with the tap water irrigation treatment in both seasons. While all tested foliar spraying of salicylic acid treatments significantly increased volume of roots, fresh and dry weights of roots compared with the control treatment in both seasons. Foliar spraying of SA at 100 and 200 ppm gave the highest values of volume of roots, fresh and dry weights of roots. In most cases, under the same saline water irrigation level, spraying SA alleviated the effect of saline water irrigation on the plants and enhance the plant growth as compared with control treatment in both seasons. The SA spraying treatments which gave the highest alleviation of the effect of saline water irrigation on plants were 100 and 200 ppm. Furthermore, the interactive effect of saline water irrigation and foliar spraying of SA which gave the highest significant value for volume of roots, fresh and dry weights of roots were obtained from the application of spraying SA 100 and 200 ppm with tap water irrigation treatment in both seasons.

\section{Flowering parameters}

Data in Table (4) show that all tested saline water irrigation treatments significantly decreased all flowering parameters compared with the irrigation by tap water treatment in both seasons. While all tested foliar spraying of salicylic acid treatments significantly increased all flowering parameters compared with the control treatment in both seasons. Foliar spraying of SA at 100 and 200 ppm gave the highest values of number of inflorescences/plant, fresh and dry weights of inflorescences, while foliar spraying of SA at $300 \mathrm{ppm}$ gave the highest value of diameter of inflorescence. In most cases, under the same saline water irrigation level, spraying SA alleviated the salinity effect on the plants and enhance the flowering parameters as compared with control treatment in both seasons. In addition, there was interactive effect of salinity and foliar spraying of SA on number of inflorescences/plant, diameter of inflorescence and fresh and dry weights of inflorescences, the highest significant values of number of inflorescences/plant and fresh and dry weights of inflorescences in both seasons were obtained by 
Table 1. Effect of irrigation by saline water and foliar spraying of salicylic acid on plant height, number of leaves/plant, leaf area and number of branches/plant of Calendula officinalis L. plant in 2014/2015 and 2015/2016 seasons

\begin{tabular}{|c|c|c|c|c|c|c|c|c|c|}
\hline \multirow{2}{*}{\multicolumn{2}{|c|}{ Treatments }} & \multicolumn{2}{|c|}{ Plant height (cm) } & \multicolumn{2}{|c|}{$\begin{array}{c}\text { Number of leaves / } \\
\text { plant }\end{array}$} & \multicolumn{2}{|c|}{ Leaf area $\left(\mathrm{cm}^{2}\right)$} & \multicolumn{2}{|c|}{$\begin{array}{c}\text { Number of branches / } \\
\text { plant }\end{array}$} \\
\hline & & $\begin{array}{c}1^{\text {st }} \\
\text { Season }\end{array}$ & $\begin{array}{c}2^{\text {nd }} \\
\text { Season }\end{array}$ & $\begin{array}{c}1^{\text {st }} \\
\text { Season }\end{array}$ & $\begin{array}{c}2^{\text {nd }} \\
\text { Season }\end{array}$ & $\begin{array}{c}1^{\text {st }} \\
\text { Season }\end{array}$ & $\begin{array}{c}2^{\text {nd }} \\
\text { Season }\end{array}$ & $\begin{array}{c}1^{\text {st }} \\
\text { Season }\end{array}$ & $\begin{array}{c}2^{\text {nd }} \\
\text { Season }\end{array}$ \\
\hline \multicolumn{10}{|c|}{ Salinity level (ppm) } \\
\hline \multicolumn{2}{|c|}{ Control ( 300 ) } & $30.25 \mathrm{a}$ & $30.67 \mathrm{a}$ & $65.83 \mathrm{a}$ & $81.00 \mathrm{a}$ & $21.41 \mathrm{a}$ & $21.71 \mathrm{a}$ & $5.33 \mathrm{a}$ & $6.25 \mathrm{a}$ \\
\hline \multicolumn{2}{|c|}{2000} & $28.92 a b$ & $27.75 b$ & $60.42 \mathrm{~b}$ & $66.00 \mathrm{~b}$ & $20.25 b$ & $20.59 \mathrm{~b}$ & $4.67 \mathrm{~b}$ & $5.42 \mathrm{~b}$ \\
\hline \multicolumn{2}{|c|}{4000} & $27.50 \mathrm{bc}$ & $24.83 \mathrm{c}$ & $55.67 \mathrm{c}$ & $55.42 \mathrm{c}$ & $19.53 \mathrm{c}$ & $20.13 b$ & $4.25 b c$ & $4.25 \mathrm{c}$ \\
\hline \multicolumn{2}{|c|}{6000} & $26.50 \mathrm{c}$ & $22.17 \mathrm{~d}$ & $50.75 d$ & $47.00 \mathrm{~d}$ & $19.03 \mathrm{~cd}$ & $19.30 \mathrm{c}$ & $3.92 \mathrm{~cd}$ & $3.92 \mathrm{c}$ \\
\hline \multicolumn{2}{|c|}{8000} & $23.17 \mathrm{~d}$ & $19.42 \mathrm{e}$ & 44.67 e & $38.08 \mathrm{e}$ & $18.49 \mathrm{~d}$ & $18.46 \mathrm{~d}$ & $3.58 d$ & $3.42 \mathrm{~d}$ \\
\hline \multicolumn{2}{|c|}{10000} & $17.92 \mathrm{e}$ & $16.08 \mathrm{f}$ & $35.75 f$ & $31.42 \mathrm{f}$ & $17.90 \mathrm{e}$ & $17.72 \mathrm{e}$ & $2.92 \mathrm{e}$ & $3.00 \mathrm{~d}$ \\
\hline \multicolumn{10}{|c|}{ SA concentration (ppm) } \\
\hline \multicolumn{2}{|c|}{ Control (0) } & $23.33 \mathrm{c}$ & $22.17 \mathrm{c}$ & $48.22 \mathrm{c}$ & $49.17 \mathrm{c}$ & $18.92 \mathrm{~b}$ & $19.20 \mathrm{~b}$ & $3.56 \mathrm{c}$ & $3.61 \mathrm{c}$ \\
\hline \multicolumn{2}{|c|}{100} & $27.50 \mathrm{a}$ & $24.72 \mathrm{a}$ & $55.83 \mathrm{a}$ & $56.89 \mathrm{a}$ & $19.62 \mathrm{a}$ & 19.69 a & $4.94 \mathrm{a}$ & $5.00 \mathrm{a}$ \\
\hline \multicolumn{2}{|c|}{200} & $26.28 a b$ & $23.94 a b$ & $53.28 \mathrm{ab}$ & $54.33 \mathrm{ab}$ & $19.55 \mathrm{a}$ & $19.96 \mathrm{a}$ & $4.11 \mathrm{~b}$ & $4.78 \mathrm{a}$ \\
\hline \multicolumn{2}{|c|}{300} & $25.72 b$ & $23.11 \mathrm{bc}$ & $51.39 \mathrm{~b}$ & 52.22 bc & $19.64 \mathrm{a}$ & $19.76 \mathrm{a}$ & $3.83 b c$ & $4.11 \mathrm{~b}$ \\
\hline \multicolumn{10}{|c|}{ Salinity * SA } \\
\hline \multirow{4}{*}{ Control } & 0 & $26.33 \mathrm{~d}-\mathrm{h}$ & $30.00 \mathrm{ab}$ & $58.67 \mathrm{~b}-\mathrm{e}$ & $76.67 \mathrm{a}$ & 20.93 a-d & 21.14 a-d & $4.67 \mathrm{c}-\mathrm{f}$ & $5.00 \mathrm{~b}-\mathrm{d}$ \\
\hline & 100 & 32.67 a & $30.33 a b$ & 70.33 a & $85.00 \mathrm{a}$ & $22.05 \mathrm{a}$ & $21.93 a b$ & $6.33 \mathrm{a}$ & $7.00 \mathrm{a}$ \\
\hline & 200 & $31.33 \mathrm{a}-\mathrm{c}$ & $31.33 \mathrm{a}$ & $71.33 \mathrm{a}$ & $84.00 \mathrm{a}$ & $21.43 a b$ & $22.22 \mathrm{a}$ & $5.67 \mathrm{ab}$ & $7.33 \mathrm{a}$ \\
\hline & 300 & $30.67 \mathrm{a}-\mathrm{c}$ & $31.00 \mathrm{a}$ & $63.00 \mathrm{bc}$ & $78.33 \mathrm{a}$ & $21.22 \mathrm{a}-\mathrm{c}$ & $21.55 \mathrm{a}-\mathrm{c}$ & $4.67 \mathrm{c}-\mathrm{f}$ & $5.67 \mathrm{bc}$ \\
\hline \multirow{4}{*}{2000} & 0 & $25.00 \mathrm{f}-\mathrm{h}$ & $25.00 \mathrm{~d}$ & $57.00 c-f$ & $62.67 \mathrm{bc}$ & 19.95 c-h & $20.28 \mathrm{de}$ & 4.00 e-h & 4.67 c-e \\
\hline & 100 & $32.00 \mathrm{ab}$ & $28.67 a b$ & $65.00 \mathrm{ab}$ & $67.67 \mathrm{~b}$ & 20.54 b-e & $20.78 b-e$ & $5.67 \mathrm{a}-\mathrm{c}$ & $6.00 \mathrm{~b}$ \\
\hline & 200 & 29.00 b-e & $29.33 a b$ & $61.33 \mathrm{~b}-\mathrm{d}$ & $68.33 \mathrm{~b}$ & 20.17 b-g & 20.84 b-e & $4.67 \mathrm{a}-\mathrm{c}$ & $6.00 \mathrm{~b}$ \\
\hline & 300 & 29.67 a-d & $28.00 \mathrm{bc}$ & $58.33 \mathrm{~b}-\mathrm{e}$ & $65.33 \mathrm{bc}$ & $20.33 b-f$ & $20.48 c-e$ & $4.33 \mathrm{~d}-\mathrm{g}$ & $5.00 \mathrm{~b}-\mathrm{d}$ \\
\hline \multirow{4}{*}{4000} & 0 & 25.67 e-h & $23.67 \mathrm{de}$ & $54.00 \mathrm{~d}-\mathrm{g}$ & $50.00 \mathrm{~d}-\mathrm{g}$ & $19.01 \mathrm{f}-\mathrm{k}$ & $19.78 \mathrm{e}-\mathrm{g}$ & $3.67 \mathrm{f}-\mathrm{i}$ & 3.67 e-h \\
\hline & 100 & 30.67 a-c & $26.00 \mathrm{~cd}$ & 58.67 b-e & $58.00 \mathrm{~cd}$ & $19.74 \mathrm{~d}-\mathrm{i}$ & 20.37 c-e & $5.00 \mathrm{c}-\mathrm{e}$ & $5.00 \mathrm{~b}-\mathrm{d}$ \\
\hline & 200 & $28.00 \mathrm{c}-\mathrm{f}$ & $26.00 \mathrm{~cd}$ & $54.00 \mathrm{~d}-\mathrm{g}$ & $56.33 \mathrm{c}-\mathrm{e}$ & $19.79 d-h$ & $20.11 d-f$ & $4.33 \mathrm{~b}-\mathrm{d}$ & $4.33 \mathrm{~d}-\mathrm{f}$ \\
\hline & 300 & 25.67 e-h & 23.67 de & $56.00 \mathrm{c}-\mathrm{g}$ & $57.33 \mathrm{~cd}$ & $19.58 \mathrm{e}-\mathrm{i}$ & 20.25 de & 4.00 e-h & $4.00 \mathrm{~d}-\mathrm{g}$ \\
\hline \multirow{4}{*}{6000} & 0 & $24.67 \mathrm{f}-\mathrm{h}$ & $20.67 \mathrm{fg}$ & $49.00 \mathrm{~g}-\mathrm{i}$ & $42.00 \mathrm{~g}-\mathrm{i}$ & $18.93 \mathrm{~g}-\mathrm{k}$ & $18.76 \mathrm{~g}-\mathrm{i}$ & $3.67 \mathrm{f}-\mathrm{i}$ & $3.00 \mathrm{gh}$ \\
\hline & 100 & $27.00 \mathrm{~d}-\mathrm{g}$ & $25.33 d$ & $53.33 \mathrm{~d}-\mathrm{h}$ & $52.33 d-f$ & 18.75 h-I & $19.01 \mathrm{f}-\mathrm{h}$ & $4.67 \mathrm{c}-\mathrm{f}$ & $4.67 \mathrm{c}-\mathrm{e}$ \\
\hline & 200 & $28.00 \mathrm{c}-\mathrm{f}$ & 22.00 ef & $48.67 \mathrm{~g}-\mathrm{i}$ & $48.00 \mathrm{e}-\mathrm{g}$ & $19.03 \mathrm{f}-\mathrm{k}$ & $19.72 \mathrm{e}-\mathrm{g}$ & $3.67 \mathrm{f}-\mathrm{i}$ & $4.33 \mathrm{~d}-\mathrm{f}$ \\
\hline & 300 & $26.33 \mathrm{~d}-\mathrm{h}$ & $20.67 \mathrm{fg}$ & $52.00 \mathrm{e}-\mathrm{h}$ & $45.67 \mathrm{f}-\mathrm{h}$ & 19.39 e-j & $19.72 \mathrm{e}-\mathrm{g}$ & $3.67 \mathrm{f}-\mathrm{i}$ & $3.67 \mathrm{e}-\mathrm{h}$ \\
\hline \multirow{4}{*}{8000} & 0 & $20.67 \mathrm{i}-\mathrm{k}$ & $19.00 \mathrm{gh}$ & 41.00 jk & $33.67 \mathrm{ij}$ & $17.52 \mathrm{Im}$ & $17.87 \mathrm{~h}-\mathrm{j}$ & $3.00 \mathrm{~h}-\mathrm{j}$ & $2.67 \mathrm{~h}$ \\
\hline & 100 & 25.67 e-h & $20.67 \mathrm{fg}$ & $49.33 \mathrm{f}-\mathrm{i}$ & $45.00 \mathrm{f}-\mathrm{h}$ & $18.44 \mathrm{i}-\mathrm{m}$ & $18.08 \mathrm{~h}-\mathrm{j}$ & $4.33 \mathrm{~d}-\mathrm{g}$ & $4.00 \mathrm{~d}-\mathrm{g}$ \\
\hline & 200 & $23.67 \mathrm{gi}$ & $18.67 \mathrm{~g}-\mathrm{i}$ & $45.67 \mathrm{~h}-\mathrm{j}$ & $38.33 \mathrm{~h}-\mathrm{j}$ & $18.70 \mathrm{~h}-\mathrm{I}$ & $19.03 \mathrm{f}-\mathrm{h}$ & $3.33 \mathrm{~g}-\mathrm{i}$ & 3.67 e-h \\
\hline & 300 & $22.67 \mathrm{~h}-\mathrm{j}$ & $19.33 \mathrm{gh}$ & $42.67 \mathrm{i}-\mathrm{k}$ & $35.33 \mathrm{ij}$ & 19.29 e-k & $18.86 \mathrm{~g}-\mathrm{i}$ & $3.67 \mathrm{f}-\mathrm{i}$ & $3.33 \mathrm{f}-\mathrm{h}$ \\
\hline \multirow{4}{*}{10000} & 0 & $17.67 \mathrm{kl}$ & $14.67 \mathrm{k}$ & $29.67 \mid$ & $30.00 \mathrm{j}$ & $17.21 \mathrm{~m}$ & $17.36 \mathrm{j}$ & $2.33 \mathrm{j}$ & $2.67 \mathrm{~h}$ \\
\hline & 100 & $17.00 \mathrm{I}$ & $17.33 \mathrm{~h}-\mathrm{j}$ & $38.33 \mathrm{jk}$ & $33.33 \mathrm{ij}$ & $18.19 \mathrm{j}-\mathrm{m}$ & 17.96 h-j & $3.67 \mathrm{f}-\mathrm{i}$ & $3.33 \mathrm{f}-\mathrm{h}$ \\
\hline & 200 & $17.67 \mathrm{kl}$ & $16.33 \mathrm{i}-\mathrm{k}$ & $38.67 \mathrm{jk}$ & $31.00 \mathrm{j}$ & $18.17 \mathrm{j}-\mathrm{m}$ & $17.83 \mathrm{~h}-\mathrm{j}$ & $3.00 \mathrm{~g}-\mathrm{i}$ & $3.00 \mathrm{gh}$ \\
\hline & 300 & $19.33 \mathrm{j}-\mathrm{I}$ & $16.00 \mathrm{jk}$ & $36.33 \mathrm{kl}$ & $31.33 \mathrm{j}$ & $18.03 \mathrm{k}-\mathrm{m}$ & $17.71 \mathrm{ij}$ & $2.67 \mathrm{ij}$ & $3.00 \mathrm{gh}$ \\
\hline
\end{tabular}

Means within a column in the same group followed by the same letter are not significantly different $(P=0.05)$ according to Duncan's multiple range test 
Effect of Saline Water Irrigation and Foliar Spraying of Salicylic Acid on Growth,

Table 2. Effect of irrigation by saline water and foliar spraying of salicylic acid on stem diameter, fresh and dry weights of the vegetative growth of Calendula officinalis L. plant in 2014/2015 and 2015/2016 seasons

\begin{tabular}{|c|c|c|c|c|c|c|c|}
\hline \multirow{2}{*}{\multicolumn{2}{|c|}{ Treatments }} & \multicolumn{2}{|c|}{ Stem diameter $(\mathbf{c m})$} & \multicolumn{2}{|c|}{$\begin{array}{c}\text { Fresh weight of } \\
\text { vegetative growth }(\mathrm{g})\end{array}$} & \multicolumn{2}{|c|}{$\begin{array}{c}\text { Dry weight of } \\
\text { vegetative growth }(\mathrm{g})\end{array}$} \\
\hline & & $\begin{array}{c}1^{\text {st }} \\
\text { Season }\end{array}$ & $\begin{array}{c}2^{\text {nd }} \\
\text { Season }\end{array}$ & $\begin{array}{c}1^{\text {st }} \\
\text { Season }\end{array}$ & $\begin{array}{l}2^{\text {nd }} \\
\text { Season }\end{array}$ & $\begin{array}{c}1^{\text {st }} \\
\text { Season }\end{array}$ & $\begin{array}{l}2^{\text {nd }} \\
\text { Season }\end{array}$ \\
\hline \multicolumn{8}{|c|}{ Salinity level (ppm) } \\
\hline \multicolumn{2}{|c|}{ Control ( 300) } & $0.52 \mathrm{a}$ & $0.53 \mathrm{a}$ & $82.08 \mathrm{a}$ & $84.32 \mathrm{a}$ & $13.28 \mathrm{a}$ & $14.16 \mathrm{a}$ \\
\hline \multirow{2}{*}{\multicolumn{2}{|c|}{$\begin{array}{l}2000 \\
4000\end{array}$}} & $0.47 \mathrm{~b}$ & $0.43 \mathrm{~b}$ & $71.51 \mathrm{~b}$ & $76.69 \mathrm{~b}$ & $12.21 \mathrm{~b}$ & $13.19 \mathrm{~b}$ \\
\hline & & $0.43 c$ & $0.41 \mathrm{bc}$ & $66.49 \mathrm{c}$ & $71.21 \mathrm{c}$ & $11.50 \mathrm{c}$ & $12.36 \mathrm{c}$ \\
\hline \multicolumn{2}{|c|}{6000} & $0.41 \mathrm{c}$ & $0.38 \mathrm{~cd}$ & $59.67 d$ & $66.22 \mathrm{~d}$ & $10.62 \mathrm{~d}$ & $11.80 \mathrm{~d}$ \\
\hline \multicolumn{2}{|c|}{8000} & $0.38 d$ & $0.36 \mathrm{~d}$ & $52.71 \mathrm{e}$ & $59.97 \mathrm{e}$ & $9.60 \mathrm{e}$ & $10.73 \mathrm{e}$ \\
\hline \multicolumn{2}{|c|}{10000} & $0.34 \mathrm{e}$ & $0.32 \mathrm{e}$ & $46.76 \mathrm{f}$ & $48.73 \mathrm{f}$ & $8.93 \mathrm{f}$ & $9.14 \mathrm{f}$ \\
\hline \multicolumn{8}{|c|}{ SA concentration (ppm) } \\
\hline \multirow{2}{*}{\multicolumn{2}{|c|}{$\begin{array}{c}\text { Control (0) } \\
100\end{array}$}} & $0.38 \mathrm{c}$ & $0.37 \mathrm{c}$ & $55.14 \mathrm{c}$ & $62.25 \mathrm{c}$ & $9.67 \mathrm{c}$ & $10.95 b$ \\
\hline & & $0.46 \mathrm{a}$ & $0.45 \mathrm{a}$ & $68.69 \mathrm{a}$ & $71.83 \mathrm{a}$ & $11.92 \mathrm{a}$ & $12.42 \mathrm{a}$ \\
\hline \multicolumn{2}{|c|}{200} & $0.44 \mathrm{~b}$ & $0.41 \mathrm{~b}$ & $67.14 \mathrm{a}$ & $69.88 \mathrm{ab}$ & $11.63 \mathrm{a}$ & $12.17 \mathrm{a}$ \\
\hline \multicolumn{2}{|c|}{300} & $0.42 \mathrm{~b}$ & $0.39 \mathrm{bc}$ & $61.85 b$ & $67.45 \mathrm{~b}$ & $10.87 b$ & $12.05 \mathrm{a}$ \\
\hline \multicolumn{8}{|c|}{ Salinity * SA } \\
\hline \multirow{4}{*}{ Control } & 0 & $0.46 \mathrm{~d}-\mathrm{f}$ & $0.44 \mathrm{c}-\mathrm{f}$ & $76.73 \mathrm{bc}$ & 80.64 a-e & $12.60 \mathrm{~cd}$ & $13.76 \mathrm{~b}$ \\
\hline & 100 & $0.58 \mathrm{a}$ & $0.63 \mathrm{a}$ & 87.73 a & 89.23 a & $13.96 \mathrm{a}$ & $15.06 \mathrm{a}$ \\
\hline & 200 & $0.53 a b$ & $0.55 b$ & $85.28 \mathrm{a}$ & $85.65 a b$ & $13.62 \mathrm{ab}$ & $14.08 \mathrm{ab}$ \\
\hline & 300 & $0.52 \mathrm{bc}$ & $0.51 \mathrm{bc}$ & $78.58 b$ & $81.74 \mathrm{a}-\mathrm{c}$ & $12.96 \mathrm{bc}$ & $13.74 b$ \\
\hline \multirow{4}{*}{2000} & 0 & $0.42 \mathrm{e}-\mathrm{g}$ & $0.41 \mathrm{~d}-\mathrm{g}$ & $63.53 \mathrm{hi}$ & $71.90 \mathrm{e}-\mathrm{g}$ & 11.09 e-h & $12.43 \mathrm{c}-\mathrm{e}$ \\
\hline & 100 & $0.50 \mathrm{~b}-\mathrm{d}$ & $0.46 \mathrm{c}-\mathrm{e}$ & $75.03 \mathrm{~b}-\mathrm{d}$ & $81.50 \mathrm{a}-\mathrm{d}$ & 13.03 bc & $13.93 \mathrm{~b}$ \\
\hline & 200 & $0.47 c-f$ & $0.37 \mathrm{f}-\mathrm{j}$ & 77.35 bc & 79.82 b-e & $13.01 \mathrm{bc}$ & $13.30 \mathrm{bc}$ \\
\hline & 300 & $0.48 \mathrm{~b}-\mathrm{e}$ & $0.48 \mathrm{~b}-\mathrm{d}$ & $70.14 \mathrm{~d}-\mathrm{f}$ & $73.54 \mathrm{c}-\mathrm{g}$ & $11.73 \mathrm{~d}-\mathrm{f}$ & $13.10 b-d$ \\
\hline \multirow{4}{*}{4000} & 0 & $0.37 \mathrm{~g}-\mathrm{i}$ & $0.40 \mathrm{~d}-\mathrm{h}$ & $58.77 \mathrm{ij}$ & $66.00 \mathrm{~g}-\mathrm{j}$ & $10.46 \mathrm{~g}-\mathrm{j}$ & $11.55 \mathrm{e}-\mathrm{h}$ \\
\hline & 100 & $0.47 \mathrm{~b}-\mathrm{f}$ & $0.41 \mathrm{~d}-\mathrm{g}$ & $71.95 \mathrm{c}-\mathrm{e}$ & $76.37 c-f$ & $12.39 \mathrm{~cd}$ & $13.02 b-d$ \\
\hline & 200 & $0.47 c-f$ & $0.44 c-f$ & $69.77 \mathrm{~d}-\mathrm{g}$ & $72.69 \mathrm{~d}-\mathrm{g}$ & $12.17 \mathrm{~cd}$ & $12.39 \mathrm{c}-\mathrm{e}$ \\
\hline & 300 & $0.42 \mathrm{f}-\mathrm{h}$ & $0.40 \mathrm{~d}-\mathrm{h}$ & $65.45 \mathrm{f}-\mathrm{h}$ & $69.78 \mathrm{f}-\mathrm{h}$ & $10.99 \mathrm{f}-\mathrm{h}$ & $12.48 \mathrm{c}-\mathrm{e}$ \\
\hline \multirow{4}{*}{6000} & 0 & $0.37 \mathrm{~g}-\mathrm{j}$ & $0.35 \mathrm{~g}-\mathrm{k}$ & $49.23 \mathrm{Im}$ & $61.64 \mathrm{~h}-\mathrm{k}$ & 8.811 & $10.91 \mathrm{f}-\mathrm{i}$ \\
\hline & 100 & $0.44 \mathrm{~d}-\mathrm{f}$ & $0.41 \mathrm{~d}-\mathrm{g}$ & 68.69 e-h & $68.90 \mathrm{f}-\mathrm{i}$ & $11.92 \mathrm{de}$ & $12.08 \mathrm{~d}-\mathrm{f}$ \\
\hline & 200 & $0.42 \mathrm{e}-\mathrm{g}$ & $0.40 d-h$ & $64.28 \mathrm{gh}$ & $69.23 \mathrm{f}-\mathrm{i}$ & $11.26 \mathrm{e}-\mathrm{g}$ & $12.51 \mathrm{c}-\mathrm{e}$ \\
\hline & 300 & $0.41 \mathrm{f}-\mathrm{h}$ & $0.37 \mathrm{e}-\mathrm{j}$ & $56.47 \mathrm{jk}$ & $65.10 \mathrm{~g}-\mathrm{j}$ & $10.47 \mathrm{~g}-\mathrm{j}$ & $11.69 \mathrm{e}-\mathrm{g}$ \\
\hline \multirow{4}{*}{8000} & 0 & $0.32 \mathrm{ij}$ & $0.32 \mathrm{~h}-\mathrm{k}$ & 43.75 no & $54.71 \mathrm{k}-\mathrm{m}$ & $7.57 \mathrm{~m}$ & $9.88 \mathrm{ij}$ \\
\hline & 100 & $0.42 \mathrm{e}-\mathrm{g}$ & 0.40 d-h & 57.32 j & $65.62 \mathrm{~g}-\mathrm{j}$ & $10.58 \mathrm{~g}-\mathrm{i}$ & $11.32 \mathrm{e}-\mathrm{h}$ \\
\hline & 200 & $0.41 \mathrm{f}-\mathrm{h}$ & $0.39 \mathrm{e}-\mathrm{i}$ & $56.58 \mathrm{jk}$ & $60.43 \mathrm{i}-\mathrm{k}$ & 10.25 h-k & $10.92 \mathrm{f}-\mathrm{i}$ \\
\hline & 300 & $0.35 \mathrm{~h}-\mathrm{j}$ & $0.32 \mathrm{~h}-\mathrm{k}$ & $53.20 \mathrm{j}-1$ & $59.14 \mathrm{j}-\mathrm{I}$ & $9.99 \mathrm{i}-\mathrm{k}$ & $10.81 \mathrm{~g}-\mathrm{i}$ \\
\hline \multirow{4}{*}{10000} & 0 & $0.31 \mathrm{j}$ & $0.29 \mathrm{jk}$ & 38.790 & $38.65 n$ & $7.49 \mathrm{~m}$ & $7.14 \mathrm{k}$ \\
\hline & 100 & $0.37 \mathrm{~g}-\mathrm{j}$ & $0.38 \mathrm{e}-\mathrm{i}$ & $51.43 \mathrm{k}-\mathrm{m}$ & $49.37 \mathrm{~m}$ & $9.63 \mathrm{j}-1$ & $9.12 \mathrm{j}$ \\
\hline & 200 & $0.33 \mathrm{ij}$ & $0.31 \mathrm{i}-\mathrm{k}$ & $49.58 \mathrm{~lm}$ & $51.48 \mathrm{Im}$ & $9.50 \mathrm{kl}$ & $9.83 \mathrm{ij}$ \\
\hline & 300 & $0.33 \mathrm{ij}$ & $0.28 \mathrm{k}$ & $47.25 \mathrm{mn}$ & $55.43 \mathrm{k}-\mathrm{m}$ & 9.091 & $10.47 \mathrm{hi}$ \\
\hline
\end{tabular}

Means within a column in the same group followed by the same letter are not significantly different $(P=0.05)$ according to Duncan's multiple range test 
Table 3. Effect of irrigation by saline water and foliar spraying of salicylic acid on roots fresh and dry weights and roots volume of Calendula officinalis L. plant in 2014/2015 and 2015/2016 seasons

\begin{tabular}{|c|c|c|c|c|c|c|c|}
\hline \multirow{2}{*}{\multicolumn{2}{|c|}{ Treatments }} & \multicolumn{2}{|c|}{ Fresh weight of roots $(\mathrm{g})$} & \multicolumn{2}{|c|}{ Dry weight of roots $(\mathrm{g})$} & \multicolumn{2}{|c|}{ Volume of roots $\left(\mathrm{cm}^{3}\right)$} \\
\hline & & $\begin{array}{c}1^{\text {st }} \\
\text { Season }\end{array}$ & $\begin{array}{c}\text { 2nd } \\
\text { Season }\end{array}$ & $\begin{array}{c}1^{\text {st }} \\
\text { Season }\end{array}$ & $\begin{array}{c}\text { 2nd } \\
\text { Season }\end{array}$ & $\begin{array}{c}1^{\text {st }} \\
\text { Season }\end{array}$ & $\begin{array}{c}\text { 2nd } \\
\text { Season }\end{array}$ \\
\hline \multicolumn{8}{|c|}{ Salinity level (ppm) } \\
\hline \multirow{2}{*}{\multicolumn{2}{|c|}{$\begin{array}{c}\text { Control ( } 300) \\
2000\end{array}$}} & $8.739 \mathrm{a}$ & $10.38 \mathrm{a}$ & $1.441 \mathrm{a}$ & $1.69 \mathrm{a}$ & $7.31 \mathrm{a}$ & $6.33 \mathrm{a}$ \\
\hline & & $6.415 b$ & $7.30 \mathrm{~b}$ & $1.159 \mathrm{~b}$ & $1.28 \mathrm{~b}$ & $6.17 \mathrm{~b}$ & $5.50 \mathrm{~b}$ \\
\hline \multicolumn{2}{|c|}{4000} & $4.620 \mathrm{c}$ & $5.80 \mathrm{c}$ & $0.877 c$ & $1.09 \mathrm{c}$ & $5.17 \mathrm{c}$ & $4.67 \mathrm{c}$ \\
\hline \multicolumn{2}{|c|}{6000} & $3.709 \mathrm{~cd}$ & $5.01 \mathrm{~d}$ & $0.696 d$ & $0.96 \mathrm{~d}$ & $4.29 \mathrm{~d}$ & $3.75 \mathrm{~d}$ \\
\hline \multicolumn{2}{|c|}{8000} & $2.791 \mathrm{de}$ & $3.76 \mathrm{e}$ & $0.544 \mathrm{de}$ & 0.73 e & $2.96 \mathrm{e}$ & $2.92 \mathrm{e}$ \\
\hline \multicolumn{2}{|c|}{10000} & $2.408 \mathrm{e}$ & $3.35 \mathrm{e}$ & $0.490 \mathrm{e}$ & $0.69 \mathrm{e}$ & $2.29 \mathrm{f}$ & $2.25 \mathrm{f}$ \\
\hline \multicolumn{8}{|c|}{ SA concentration (ppm) } \\
\hline \multicolumn{2}{|c|}{ Control (0) } & $3.642 \mathrm{c}$ & $5.26 \mathrm{~b}$ & $0.670 \mathrm{c}$ & $0.94 \mathrm{~b}$ & $3.70 \mathrm{c}$ & $3.36 \mathrm{c}$ \\
\hline \multicolumn{2}{|c|}{100} & $5.576 \mathrm{a}$ & $6.53 \mathrm{a}$ & $1.017 \mathrm{a}$ & $1.17 \mathrm{a}$ & $5.61 \mathrm{a}$ & $5.03 \mathrm{a}$ \\
\hline \multicolumn{2}{|c|}{200} & $5.366 a b$ & $6.02 \mathrm{a}$ & $0.964 \mathrm{a}$ & $1.09 \mathrm{a}$ & $5.25 \mathrm{a}$ & $4.75 \mathrm{a}$ \\
\hline \multicolumn{2}{|c|}{300} & $4.538 \mathrm{~b}$ & $5.92 \mathrm{a}$ & $0.819 b$ & $1.09 \mathrm{a}$ & $4.22 \mathrm{~b}$ & $3.81 \mathrm{~b}$ \\
\hline \multicolumn{8}{|c|}{ Salinity * SA } \\
\hline \multirow{4}{*}{ Control } & 0 & $6.400 \mathrm{c}-\mathrm{f}$ & $9.17 \mathrm{~b}$ & $1.131 \mathrm{c}-\mathrm{e}$ & $1.46 \mathrm{~cd}$ & $5.89 c-e$ & $5.17 \mathrm{~cd}$ \\
\hline & 100 & $9.585 \mathrm{~b}$ & $11.68 \mathrm{a}$ & $1.578 \mathrm{ab}$ & $1.90 \mathrm{a}$ & $8.50 \mathrm{a}$ & $7.00 \mathrm{a}$ \\
\hline & 200 & $11.750 \mathrm{a}$ & $11.45 \mathrm{a}$ & $1.890 \mathrm{a}$ & $1.80 \mathrm{ab}$ & $7.50 \mathrm{ab}$ & $6.83 \mathrm{a}$ \\
\hline & 300 & $7.220 \mathrm{~cd}$ & $9.23 \mathrm{~b}$ & $1.164 \mathrm{c}-\mathrm{e}$ & $1.60 \mathrm{bc}$ & $7.33 a b$ & $6.33 a b$ \\
\hline \multirow{4}{*}{2000} & 0 & $4.650 \mathrm{e}-\mathrm{j}$ & $6.95 \mathrm{c}-\mathrm{e}$ & 0.849 e-h & 1.16 ef & $4.67 \mathrm{e}-\mathrm{h}$ & $4.50 \mathrm{~d}-\mathrm{g}$ \\
\hline & F100 & $8.460 \mathrm{bc}$ & $7.37 \mathrm{~cd}$ & 1.478 bc & $1.26 \mathrm{de}$ & $7.50 a b$ & $6.50 \mathrm{a}$ \\
\hline & 200 & $7.060 \mathrm{c}-\mathrm{e}$ & $6.84 \mathrm{c}-\mathrm{e}$ & $1.307 \mathrm{bd}$ & $1.28 \mathrm{de}$ & $7.00 \mathrm{bc}$ & $6.17 \mathrm{ab}$ \\
\hline & 300 & $5.490 \mathrm{~d}-\mathrm{g}$ & $8.06 \mathrm{bc}$ & $1.002 \mathrm{~d}-\mathrm{f}$ & $1.42 \mathrm{~cd}$ & $5.50 \mathrm{~d}-\mathrm{f}$ & $4.83 c-f$ \\
\hline \multirow{4}{*}{4000} & 0 & $4.000 \mathrm{f}-\mathrm{I}$ & $5.13 \mathrm{f}-\mathrm{j}$ & $0.750 \mathrm{e}-\mathrm{i}$ & $0.93 \mathrm{f}-\mathrm{h}$ & $4.33 \mathrm{f}-\mathrm{i}$ & $4.00 \mathrm{f}-\mathrm{h}$ \\
\hline & 100 & $4.945 \mathrm{~d}-\mathrm{i}$ & $6.55 \mathrm{c}-\mathrm{f}$ & $0.987 \mathrm{~d}-\mathrm{f}$ & $1.27 \mathrm{de}$ & $6.33 b-d$ & $5.50 \mathrm{bc}$ \\
\hline & 200 & $4.400 \mathrm{f}-\mathrm{k}$ & $5.57 \mathrm{e}-\mathrm{i}$ & $0.802 \mathrm{e}-\mathrm{h}$ & $1.05 \mathrm{e}-\mathrm{g}$ & $5.67 \mathrm{de}$ & $5.00 \mathrm{c}-\mathrm{e}$ \\
\hline & 300 & $5.135 d-h$ & $5.95 \mathrm{~d}-\mathrm{g}$ & $0.971 \mathrm{~d}-\mathrm{g}$ & $1.11 \mathrm{ef}$ & $4.33 \mathrm{f}-\mathrm{i}$ & $4.17 \mathrm{e}-\mathrm{h}$ \\
\hline \multirow{4}{*}{6000} & 0 & $3.050 \mathrm{~g}-\mathrm{I}$ & $4.04 \mathrm{i}-\mathrm{k}$ & $0.561 \mathrm{~g}-\mathrm{i}$ & 0.77hi & $3.17 \mathrm{i}-\mathrm{m}$ & $2.67 \mathrm{i}-\mathrm{k}$ \\
\hline & 100 & $4.160 \mathrm{f}-\mathrm{I}$ & $5.88 \mathrm{~d}-\mathrm{h}$ & $0.821 \mathrm{e}-\mathrm{h}$ & 1.11 ef & $5.00 \mathrm{e}-\mathrm{g}$ & $4.50 \mathrm{~d}-\mathrm{g}$ \\
\hline & 200 & $4.245 \mathrm{f}-\mathrm{I}$ & $5.49 \mathrm{e}-\mathrm{i}$ & $0.778 \mathrm{e}-\mathrm{i}$ & $1.05 \mathrm{e}-\mathrm{g}$ & $5.17 \mathrm{~d}-\mathrm{f}$ & $4.50 \mathrm{~d}-\mathrm{g}$ \\
\hline & 300 & $3.380 \mathrm{~g}-1$ & $4.63 \mathrm{~g}-\mathrm{k}$ & $0.623 \mathrm{f}-\mathrm{i}$ & $0.91 \mathrm{f}-\mathrm{i}$ & $3.83 \mathrm{~g}-\mathrm{j}$ & $3.33 \mathrm{hi}$ \\
\hline \multirow{4}{*}{8000} & 0 & $1.950 \mathrm{kl}$ & $3.30 \mathrm{k}$ & $0.373 \mathrm{i}$ & $0.65 \mathrm{i}$ & 2.33 I-n & $2.17 \mathrm{j}-\mathrm{I}$ \\
\hline & 100 & $3.390 \mathrm{~g}-1$ & 4.25 h-k & $0.657 \mathrm{f}-\mathrm{i}$ & $0.80 \mathrm{~g}-\mathrm{i}$ & 3.50 h-I & $3.83 \mathrm{gh}$ \\
\hline & 200 & $2.525 \mathrm{i}-\mathrm{I}$ & 3.56 jk & $0.531 \mathrm{hi}$ & $0.70 \mathrm{hi}$ & 3.67 h-k & $3.33 \mathrm{hi}$ \\
\hline & 300 & $3.300 \mathrm{~g}-1$ & $3.92 \mathrm{i}-\mathrm{k}$ & $0.614 \mathrm{f}-\mathrm{i}$ & $0.75 \mathrm{hi}$ & $2.33 \mathrm{I}-\mathrm{n}$ & $2.33 \mathrm{j}-\mathrm{I}$ \\
\hline \multirow{4}{*}{10000} & 0 & $1.800 \mathrm{I}$ & $2.98 \mathrm{k}$ & $0.357 \mathrm{i}$ & $0.66 \mathrm{i}$ & $1.83 n$ & 1.67 I \\
\hline & 100 & 2.915 h-I & $3.47 \mathrm{jk}$ & $0.581 \mathrm{f}-\mathrm{i}$ & $0.70 \mathrm{hi}$ & 2.83 j-n & $2.83 \mathrm{ij}$ \\
\hline & 200 & $2.215 \mathrm{j}-\mathrm{I}$ & $3.21 \mathrm{k}$ & 0.479 hi & $0.66 \mathrm{i}$ & $2.50 \mathrm{k}-\mathrm{n}$ & $2.67 \mathrm{i}-\mathrm{k}$ \\
\hline & 300 & $2.700 \mathrm{~h}-\mathrm{I}$ & $3.74 \mathrm{jk}$ & $0.541 \mathrm{hi}$ & 0.74 hi & $2.00 \mathrm{mn}$ & $1.83 \mathrm{kl}$ \\
\hline
\end{tabular}

Means within a column in the same group followed by the same letter are not significantly different $(P=0.05)$ according to Duncan's multiple range test 
Effect of Saline Water Irrigation and Foliar Spraying of Salicylic Acid on Growth,

Table 4. Effect of irrigation by saline water and foliar spraying of salicylic acid on number of inflorescences/plant, diameter of inflorescence, fresh and dry weights of inflorescences of Calendula officinalis L. plant in 2014/2015 and 2015/2016 seasons

\begin{tabular}{|c|c|c|c|c|c|c|c|c|c|}
\hline \multirow{2}{*}{\multicolumn{2}{|c|}{ Treatments }} & \multicolumn{2}{|c|}{$\begin{array}{c}\text { Number of } \\
\text { inflorescences / } \\
\text { plant }\end{array}$} & \multicolumn{2}{|c|}{$\begin{array}{c}\text { Diameter of } \\
\text { inflorescence }(\mathrm{cm})\end{array}$} & \multicolumn{2}{|c|}{$\begin{array}{l}\text { Fresh weight of } \\
\text { inflorescences } \\
(\mathrm{g})\end{array}$} & \multicolumn{2}{|c|}{$\begin{array}{l}\text { Dry weight of } \\
\text { inflorescences } \\
\text { (g) }\end{array}$} \\
\hline & & $\begin{array}{c}1^{\text {st }} \\
\text { Season }\end{array}$ & $\begin{array}{c}2^{\text {nd }} \\
\text { Season }\end{array}$ & $\begin{array}{c}1^{\text {st }} \\
\text { Season }\end{array}$ & $\begin{array}{c}\text { 2nd } \\
\text { Season }\end{array}$ & $\begin{array}{c}1^{\text {st }} \\
\text { Season }\end{array}$ & $\begin{array}{c}\text { 2nd } \\
\text { Season }\end{array}$ & $\begin{array}{c}1^{\text {st }} \\
\text { Season }\end{array}$ & $\begin{array}{l}\text { 2nd } \\
\text { Season }\end{array}$ \\
\hline \multicolumn{10}{|c|}{ Salinity level (ppm) } \\
\hline \multicolumn{2}{|c|}{ Control(300) } & $12.51 \mathrm{a}$ & $11.18 \mathrm{a}$ & $3.35 \mathrm{a}$ & $3.00 \mathrm{a}$ & $38.65 \mathrm{a}$ & $46.46 \mathrm{a}$ & $6.20 \mathrm{a}$ & $7.42 \mathrm{a}$ \\
\hline \multicolumn{2}{|c|}{2000} & $9.59 \mathrm{~b}$ & $9.32 \mathrm{~b}$ & $3.12 b$ & $2.91 \mathrm{~b}$ & $33.02 \mathrm{~b}$ & $37.83 \mathrm{~b}$ & $5.33 \mathrm{~b}$ & $5.84 \mathrm{~b}$ \\
\hline \multicolumn{2}{|c|}{4000} & $8.78 \mathrm{c}$ & $7.78 \mathrm{c}$ & $3.06 \mathrm{c}$ & $2.80 \mathrm{c}$ & $29.33 \mathrm{c}$ & $34.00 \mathrm{c}$ & $4.70 \mathrm{c}$ & $5.45 \mathrm{~b}$ \\
\hline \multicolumn{2}{|c|}{6000} & $7.54 \mathrm{~d}$ & $5.98 d$ & $2.87 \mathrm{~d}$ & $2.62 \mathrm{~d}$ & $24.55 \mathrm{~d}$ & $30.15 \mathrm{~d}$ & $3.95 d$ & $4.54 \mathrm{c}$ \\
\hline \multicolumn{2}{|c|}{8000} & $6.06 \mathrm{e}$ & $4.10 \mathrm{e}$ & $2.70 \mathrm{e}$ & $2.46 \mathrm{e}$ & $17.83 \mathrm{e}$ & 20.58 e & $2.87 \mathrm{e}$ & $3.60 \mathrm{~d}$ \\
\hline \multicolumn{2}{|c|}{10000} & $3.96 \mathrm{f}$ & $3.17 \mathrm{f}$ & $2.37 \mathrm{f}$ & $2.38 \mathrm{f}$ & $10.87 \mathrm{f}$ & $10.39 \mathrm{f}$ & $2.00 \mathrm{f}$ & $1.98 \mathrm{e}$ \\
\hline \multicolumn{10}{|c|}{ SA concentration (ppm) } \\
\hline \multirow{2}{*}{\multicolumn{2}{|c|}{$\begin{array}{c}\text { Control (0) } \\
100\end{array}$}} & $5.31 \mathrm{~d}$ & $5.00 \mathrm{c}$ & $2.69 \mathrm{~d}$ & $2.59 \mathrm{c}$ & $20.99 c$ & $22.22 \mathrm{c}$ & $3.38 \mathrm{c}$ & $3.75 \mathrm{c}$ \\
\hline & & $10.09 a$ & $8.20 \mathrm{a}$ & $2.77 \mathrm{c}$ & $2.69 \mathrm{~b}$ & $28.18 \mathrm{a}$ & $33.80 \mathrm{a}$ & $4.63 \mathrm{a}$ & $5.38 \mathrm{a}$ \\
\hline \multicolumn{2}{|c|}{200} & $9.85 b$ & $8.21 \mathrm{a}$ & $3.06 \mathrm{~b}$ & $2.68 \mathrm{~b}$ & $28.22 \mathrm{a}$ & $34.33 \mathrm{a}$ & $4.69 \mathrm{a}$ & $5.58 \mathrm{a}$ \\
\hline \multicolumn{2}{|l|}{300} & $7.05 \mathrm{c}$ & $6.26 \mathrm{~b}$ & $3.12 \mathrm{a}$ & $2.82 \mathrm{a}$ & $25.45 b$ & $29.25 b$ & $4.00 \mathrm{~b}$ & $4.51 \mathrm{~b}$ \\
\hline \multicolumn{10}{|c|}{ Salinity * SA } \\
\hline \multirow{4}{*}{ Control } & 0 & $9.40 \mathrm{~g}$ & $8.93 \mathrm{e}$ & $3.11 \mathrm{f}$ & $2.87 \mathrm{c}-\mathrm{e}$ & $34.89 \mathrm{~b}$ & $41.17 \mathrm{~cd}$ & $5.62 \mathrm{~cd}$ & $6.70 \mathrm{~b}-\mathrm{d}$ \\
\hline & 100 & $15.78 \mathrm{a}$ & $13.19 \mathrm{a}$ & $3.06 \mathrm{~g}$ & $3.10 a b$ & $40.93 a$ & $47.17 \mathrm{bc}$ & $6.67 \mathrm{ab}$ & $7.14 a b$ \\
\hline & 200 & $14.27 b$ & $12.61 \mathrm{ab}$ & $3.48 \mathrm{~b}$ & $2.98 a-c$ & $42.21 \mathrm{a}$ & $54.94 \mathrm{a}$ & $6.83 \mathrm{a}$ & $8.80 \mathrm{a}$ \\
\hline & 300 & $10.60 \mathrm{f}$ & $10.00 \mathrm{de}$ & $3.75 \mathrm{a}$ & $3.12 \mathrm{a}$ & $36.58 \mathrm{~b}$ & $42.55 \mathrm{~b}$ & $5.68 \mathrm{~cd}$ & $7.05 \mathrm{~b}$ \\
\hline \multirow{4}{*}{2000} & 0 & $6.75 \mathrm{j}$ & $7.25 \mathrm{f}$ & $2.80 \mathrm{j}$ & $2.75 d-f$ & $29.97 c$ & $32.29 \mathrm{e}-\mathrm{g}$ & 4.78 ef & $4.84 d-h$ \\
\hline & 100 & $11.78 \mathrm{~cd}$ & $11.41 \mathrm{bc}$ & $3.01 \mathrm{~h}$ & $2.93 a-d$ & $34.62 \mathrm{~b}$ & $40.14 \mathrm{~cd}$ & $5.62 \mathrm{~cd}$ & $6.47 \mathrm{~b}-\mathrm{e}$ \\
\hline & 200 & $12.22 \mathrm{c}$ & $11.48 \mathrm{bc}$ & $3.30 \mathrm{~d}$ & $2.92 a-d$ & $36.80 \mathrm{~b}$ & $41.01 \mathrm{~cd}$ & $6.07 \mathrm{bc}$ & $6.81 \mathrm{bc}$ \\
\hline & 300 & $7.60 \mathrm{hi}$ & $7.13 \mathrm{f}$ & $3.35 \mathrm{c}$ & $2.98 \mathrm{a}-\mathrm{c}$ & $30.70 \mathrm{c}$ & $37.86 \mathrm{c}-\mathrm{e}$ & 4.85 ef & $5.24 b-g$ \\
\hline \multirow{4}{*}{4000} & 0 & 4.87 I & $4.47 \mathrm{hi}$ & $2.70 \mathrm{i}$ & $2.67 \mathrm{e}-\mathrm{g}$ & $26.45 \mathrm{de}$ & $29.05 \mathrm{~g}$ & $4.21 \mathrm{f}-\mathrm{h}$ & $4.54 \mathrm{e}-\mathrm{i}$ \\
\hline & 100 & $11.25 \mathrm{de}$ & $9.42 \mathrm{de}$ & $2.98 \mathrm{~h}$ & $2.83 c-e$ & $30.87 \mathrm{c}$ & $37.93 \mathrm{c}-\mathrm{e}$ & $5.03 \mathrm{de}$ & $6.28 \mathrm{~b}-\mathrm{f}$ \\
\hline & 200 & 11.00 ef & $10.25 \mathrm{~cd}$ & $3.24 \mathrm{e}$ & $2.80 \mathrm{c}-\mathrm{e}$ & $31.20 \mathrm{c}$ & $36.53 c-f$ & $5.04 \mathrm{de}$ & $6.02 \mathrm{~b}-\mathrm{f}$ \\
\hline & 300 & $8.00 \mathrm{~h}$ & $7.00 \mathrm{f}$ & $3.32 \mathrm{~cd}$ & $2.90 \mathrm{~b}-\mathrm{d}$ & $28.80 \mathrm{~cd}$ & $32.47 \mathrm{e}-\mathrm{g}$ & $4.53 \mathrm{e}-\mathrm{g}$ & $4.93 \mathrm{c}-\mathrm{h}$ \\
\hline \multirow{4}{*}{6000} & 0 & 4.831 & $4.33 \mathrm{hi}$ & $2.63 \mathrm{~m}$ & $2.55 \mathrm{f}-\mathrm{h}$ & $19.19 \mathrm{f}$ & $18.30 \mathrm{hi}$ & $3.14 \mathrm{jk}$ & $3.13 \mathrm{~h}-\mathrm{j}$ \\
\hline & 100 & $9.33 \mathrm{~g}$ & $6.96 \mathrm{f}$ & $2.76 \mathrm{k}$ & $2.53 \mathrm{gh}$ & $26.93 \mathrm{de}$ & $35.16 \mathrm{~d}-\mathrm{g}$ & 4.49 e-g & $5.35 \mathrm{~b}-\mathrm{g}$ \\
\hline & 200 & $9.12 \mathrm{~g}$ & $6.61 \mathrm{f}$ & $3.20 \mathrm{e}$ & $2.58 \mathrm{f}-\mathrm{h}$ & $26.45 \mathrm{de}$ & $35.66 \mathrm{~d}-\mathrm{f}$ & $4.17 \mathrm{f}-\mathrm{h}$ & $5.30 \mathrm{~b}-\mathrm{g}$ \\
\hline & 300 & $6.89 j$ & $6.00 \mathrm{fg}$ & $2.90 \mathrm{i}$ & $2.82 \mathrm{c}-\mathrm{e}$ & $25.63 \mathrm{e}$ & $31.48 \mathrm{fg}$ & $4.01 \mathrm{~g}-\mathrm{i}$ & $4.39 \mathrm{f}-\mathrm{i}$ \\
\hline \multirow{4}{*}{8000} & 0 & $3.33 \mathrm{~m}$ & $2.67 \mathrm{j}$ & $2.60 \mathrm{mn}$ & $2.42 \mathrm{~h}-\mathrm{j}$ & $9.75 \mathrm{~h}$ & $8.42 \mathrm{jk}$ & $1.64 \mid$ & $2.38 \mathrm{jk}$ \\
\hline & 100 & $7.60 \mathrm{hi}$ & $4.67 \mathrm{hi}$ & $2.56 n$ & $2.42 \mathrm{~h}-\mathrm{j}$ & $21.20 \mathrm{f}$ & $29.35 \mathrm{~g}$ & $3.49 \mathrm{~h}-\mathrm{j}$ & $4.71 \mathrm{e}-\mathrm{i}$ \\
\hline & 200 & $7.30 \mathrm{ij}$ & $4.00 \mathrm{hi}$ & $2.83 \mathrm{j}$ & $2.42 \mathrm{~h}-\mathrm{j}$ & $19.55 \mathrm{f}$ & $22.60 \mathrm{~h}$ & $3.04 \mathrm{jk}$ & $3.71 \mathrm{~g}-\mathrm{j}$ \\
\hline & 300 & $6.00 \mathrm{k}$ & $5.05 \mathrm{gh}$ & $2.82 \mathrm{j}$ & $2.58 \mathrm{f}-\mathrm{h}$ & $20.83 \mathrm{f}$ & $21.96 \mathrm{~h}$ & $3.31 \mathrm{ij}$ & $3.61 \mathrm{~g}-\mathrm{j}$ \\
\hline \multirow{4}{*}{10000} & 0 & $2.67 n$ & $2.33 \mathrm{j}$ & 2.330 & $2.32 \mathrm{ij}$ & $5.70 \mathrm{i}$ & $4.12 \mathrm{k}$ & $0.89 \mathrm{~m}$ & $0.89 \mathrm{k}$ \\
\hline & 100 & 4.801 & $3.60 \mathrm{ij}$ & $2.23 p$ & $2.30 \mathrm{j}$ & $14.53 \mathrm{~g}$ & $13.04 \mathrm{ij}$ & $2.45 \mathrm{k}$ & $2.35 \mathrm{jk}$ \\
\hline & 200 & 5.181 & $4.33 \mathrm{hi}$ & 2.330 & $2.37 \mathrm{~h}-\mathrm{j}$ & $13.08 \mathrm{~g}$ & $15.25 \mathrm{i}$ & $2.97 \mathrm{jk}$ & $2.84 \mathrm{ij}$ \\
\hline & 300 & $3.20 \mathrm{~m}$ & $2.40 \mathrm{j}$ & $2.57 n$ & $2.52 \mathrm{~g}-\mathrm{i}$ & $10.17 \mathrm{~h}$ & $9.15 \mathrm{jk}$ & 1.621 & $1.85 \mathrm{jk}$ \\
\hline
\end{tabular}

Means within a column in the same group followed by the same letter are not significantly different $(P=0.05)$ according to Duncan's multiple range test 
the application of SA foliar sprayings at concentrations 100 and 200 ppm with tap water irrigation treatment, while the highest significant value of diameter of inflorescence was obtained by spraying SA at 300 ppm with tap water irrigation treatment.

As for the salinity and SA effects on the flowering parameters, the obtained results agree with those reported by Bayat et al (2012), Pacheco et al (2013), Hashish et al (2015a), and Nofal et al (2015) on pot marigold.

Leaf total Chlorophyll, carotene contents in flowers and proline content measurements

Data in Table (5) show that all tested saline water irrigation treatments significantly decreased carotene contents in flowers and leaf total chlorophyll, while proline content increased compared with the tap water irrigation treatment in both seasons. On the other hand, all tested foliar spraying of salicylic acid treatments significantly increased carotene contents in flowers, leaf total chlorophyll and content of proline compared with the control treatment in both seasons. Foliar spraying of SA at 100 and 200 ppm gave the highest values of flower carotenes and proline contents. In most cases, under the same saline water irrigation level, spraying SA alleviated the salinity effect on the plants and enhance the plant growth as compared with control treatment in both seasons. In addition, there was interactive effect of salinity and foliar spraying of SA on carotene contents in flowers, leaf total chlorophyll and content of proline, the highest significant values of carotene contents in flowers and leaf total chlorophyll were obtained by the application of SA foliar sprayings at concentrations 100, $200 \mathrm{ppm}$ with tap water irrigation treatment in both seasons. While, the highest significant values of proline content in both seasons were obtained by the application of SA foliar sprayings at concentrations 100, 200 ppm with 8000 and $10000 \mathrm{ppm}$ saline water irrigation treatments, and foliar spraying of SA at 300 ppm with 8000 ppm saline water irrigation treatment in both seasons.

These results coincide with those reported by Moharekar et al (2003) and Syeed (2008) who found that the total carotenes increased significantly in wheat and mungbean by increasing SA concentration. Concerning chlorophyll content in leaves, Ghai et al (2002) reported that foliar spraying of SA with concentration $(20 \mathrm{mg} / \mathrm{ml})$ on Brassica napus plants improved the chlorophyll content, while Karlidag et al (2009) mentioned that strawberry plants treated with SA exhibited greater growth, as did higher chlorophyll concentrations under salt stress. Also, Pesci, 1987, and Kuznetsov \& Shevyakova, 1999 revealed that proline accumulation is effective cell osmoprotectant; the application of exogenous SA increase the proline content in the plant cells as reported by Shakirova et al 2003 on wheat and Bandurska and Stroinski, 2005 on barley. In addition, similar findings were reported by Syeed and Khan (2010), Bayat et al (2012), and Pacheco et al (2013).

\section{Mineral analysis of leaves and survival per- centage}

Data in Tables (6 and 7) show that all tested saline water irrigation treatments significantly decreased nitrogen, phosphorus and potassium. While, calcium, sodium and chloride percentage significantly increased in both seasons. On the other hand, all tested foliar spraying of salicylic acid treatments significantly increased nitrogen, phosphorus, potassium, calcium and survival percentages compared with the control treatment in both seasons, while it decreased sodium and chloride percentages. Foliar spraying of SA at 100 and 200 ppm gave the highest values of nitrogen, phosphorus, potassium, calcium survival percentages, while it gave the lowest sodium and chloride percentages. . In most cases, under the same saline water irrigation level, spraying SA alleviated the salinity effect on the plants and enhanced the plant growth as compared with control treatment in both seasons. In addition, there was interactive effect of salinity and foliar spraying of SA on number of nitrogen, phosphorus, potassium and calcium percentages, the highest significant values of nitrogen and potassium percentages in both seasons were obtained by the application of SA foliar sprayings at 100 and $200 \mathrm{ppm}$ with control salinity treatment. While, the highest significant values of phosphorus percentage in both seasons were obtained by the application of SA foliar sprayings at 100, 200 and 300 ppm with control and 2000 ppm salinity treatments. However, the highest significant values of calcium percentage in both seasons were obtained by the application of SA foliar spraying at 200 ppm with 10000 ppm salinity treatment. Moreover, the highest significant values of survival percentage in both seasons were obtained by the application of all foliar sprayings treatments with control and 2000 ppm salinity treatments, also obtained by SA foliar spraying at 100 and 200 ppm with 4000 ppm salinity treatment in both seasons. These results agree with those reported by Bayat et al (2012), and Pacheco et al (2013). 
Effect of Saline Water Irrigation and Foliar Spraying of Salicylic Acid on Growth,

Table 5. Effect of irrigation by saline water and foliar spraying of salicylic acid on carotene contents in flowers, proline content and leaf total chlorophyll of Calendula officinalis L. plant in 2014/2015 and 2015/2016 seasons

\begin{tabular}{|c|c|c|c|c|c|c|c|}
\hline & & \multicolumn{2}{|c|}{$\begin{array}{c}\text { carotene contents in } \\
\text { flowers } \\
\text { (mg/g f. wt.) }\end{array}$} & \multicolumn{2}{|c|}{$\begin{array}{c}\text { Content of proline } \\
(\mu \mathrm{g} / \mathrm{g} \text { f.w.) }\end{array}$} & \multicolumn{2}{|c|}{$\begin{array}{l}\text { Leaf total } \\
\text { chlorophyll } \\
\text { (mg/g f. wt.) }\end{array}$} \\
\hline \multicolumn{2}{|c|}{ Treatments } & $\begin{array}{c}1^{\text {st }} \\
\text { Season }\end{array}$ & $\begin{array}{c}2^{\text {nd }} \\
\text { Season }\end{array}$ & $\begin{array}{c}1^{\text {st }} \\
\text { Season }\end{array}$ & $\begin{array}{c}2^{\text {nd }} \\
\text { Season }\end{array}$ & $\begin{array}{c}1^{\text {st }} \\
\text { Season }\end{array}$ & $\begin{array}{c}2^{\text {nd }} \\
\text { Season }\end{array}$ \\
\hline \multicolumn{8}{|c|}{ Salinity level (ppm) } \\
\hline \multicolumn{2}{|c|}{$\begin{array}{c}\text { Control(300) } \\
2000 \\
4000 \\
6000 \\
8000 \\
10000 \\
\end{array}$} & $\begin{array}{c}1.137 \mathrm{a} \\
0.953 \mathrm{~b} \\
0.865 \mathrm{c} \\
0.828 \mathrm{~cd} \\
0.782 \mathrm{~d} \\
0.711 \mathrm{e} \\
\end{array}$ & $\begin{array}{c}1.169 \mathrm{a} \\
0.944 \mathrm{~b} \\
0.854 \mathrm{bc} \\
0.822 \mathrm{c} \\
0.784 \mathrm{c} \\
0.675 \mathrm{~d} \\
\end{array}$ & $\begin{array}{c}153.41 \mathrm{e} \\
246.98 \mathrm{~d} \\
349.87 \mathrm{c} \\
528.49 \mathrm{~b} \\
639.73 \mathrm{a} \\
583.60 \mathrm{ab} \\
\end{array}$ & $\begin{array}{l}150.22 \mathrm{e} \\
240.63 \mathrm{~d} \\
344.61 \mathrm{c} \\
497.41 \mathrm{~b} \\
534.83 \mathrm{a} \\
545.55 \mathrm{a}\end{array}$ & $\begin{array}{c}0.984 \mathrm{a} \\
0.665 \mathrm{~b} \\
0.652 \mathrm{bc} \\
0.609 \mathrm{bc} \\
0.526 \mathrm{c} \\
0.294 \mathrm{~d} \\
\end{array}$ & $\begin{array}{c}1.071 \mathrm{a} \\
0.527 \mathrm{~b} \\
0.344 \mathrm{c} \\
0.268 \mathrm{~cd} \\
0.217 \mathrm{~d} \\
0.192 \mathrm{~d} \\
\end{array}$ \\
\hline \multicolumn{8}{|c|}{ SA concentration (ppm) } \\
\hline \multicolumn{2}{|c|}{$\begin{array}{c}\text { Control (0) } \\
100 \\
200 \\
300 \\
\end{array}$} & $\begin{array}{c}0.774 \mathrm{c} \\
0.911 \mathrm{ab} \\
0.949 \mathrm{a} \\
0.884 \mathrm{~b}\end{array}$ & $\begin{array}{l}0.755 \mathrm{~b} \\
0.907 \mathrm{a} \\
0.949 \mathrm{a} \\
0.888 \mathrm{a}\end{array}$ & $\begin{array}{c}309.33 \mathrm{c} \\
489.70 \mathrm{a} \\
451.52 \mathrm{ab} \\
417.50 \mathrm{~b}\end{array}$ & $\begin{array}{c}313.68 \mathrm{c} \\
410.45 \mathrm{ab} \\
430.68 \mathrm{a} \\
387.35 \mathrm{~b}\end{array}$ & $\begin{array}{l}0.489 \mathrm{~b} \\
0.658 \mathrm{a} \\
0.719 \mathrm{a} \\
0.622 \mathrm{a}\end{array}$ & $\begin{array}{l}0.353 \mathrm{~b} \\
0.482 \mathrm{a} \\
0.461 \mathrm{a} \\
0.451 \mathrm{a}\end{array}$ \\
\hline \multicolumn{8}{|c|}{ Salinity * SA } \\
\hline Control & $\begin{array}{c}0 \\
100 \\
200 \\
300\end{array}$ & $\begin{array}{l}1.080 \mathrm{a}-\mathrm{c} \\
1.178 \mathrm{a} \\
1.197 \mathrm{a} \\
1.093 \mathrm{ab}\end{array}$ & $\begin{array}{l}1.100 \mathrm{a}-\mathrm{d} \\
1.200 \mathrm{ab} \\
1.263 \mathrm{a} \\
1.114 \mathrm{a}-\mathrm{c}\end{array}$ & $\begin{array}{c}109.67 \mathrm{j} \\
181.66 \mathrm{ij} \\
207.16 \mathrm{~h}-\mathrm{j} \\
115.17 \mathrm{j} \\
\end{array}$ & $\begin{array}{l}108.56 \mathrm{l} \\
146.92 \mathrm{kl} \\
171.57 \mathrm{jk} \\
173.83 \mathrm{jk} \\
\end{array}$ & $\begin{array}{c}0.902 \mathrm{bc} \\
0.977 \mathrm{ab} \\
1.214 \mathrm{a} \\
0.843 \mathrm{~b}-\mathrm{d}\end{array}$ & $\begin{array}{r}0.911 \mathrm{~b} \\
1.167 \mathrm{a} \\
1.129 \mathrm{a} \\
1.076 \mathrm{ab}\end{array}$ \\
\hline 2000 & $\begin{array}{c}0 \\
100 \\
200 \\
300 \\
\end{array}$ & $\begin{array}{l}0.800 \mathrm{~g}-\mathrm{I} \\
1.003 \mathrm{~b}-\mathrm{e} \\
1.030 \mathrm{~b}-\mathrm{d} \\
0.979 \mathrm{~b}-\mathrm{f}\end{array}$ & $\begin{array}{l}0.742 \mathrm{~h}-\mathrm{k} \\
0.983 \mathrm{c}-\mathrm{g} \\
1.053 \mathrm{~b}-\mathrm{e} \\
0.997 \mathrm{~b}-\mathrm{f}\end{array}$ & $\begin{array}{l}180.60 \mathrm{ij} \\
303.66 \mathrm{f}-\mathrm{i} \\
236.03 \mathrm{~h}-\mathrm{j} \\
267.60 \mathrm{~g}-\mathrm{i}\end{array}$ & $\begin{array}{l}197.21 \mathrm{i}-\mathrm{k} \\
271.33 \mathrm{gh} \\
279.92 \mathrm{~g} \\
214.04 \mathrm{~h}-\mathrm{j}\end{array}$ & $\begin{array}{l}0.587 \mathrm{~d}-\mathrm{g} \\
0.684 \mathrm{~b}-\mathrm{f} \\
0.736 \mathrm{~b}-\mathrm{f} \\
0.654 \mathrm{c}-\mathrm{f}\end{array}$ & $\begin{array}{c}0.373 \mathrm{~d}-\mathrm{f} \\
0.616 \mathrm{c} \\
0.598 \mathrm{c} \\
0.523 \mathrm{~cd}\end{array}$ \\
\hline 4000 & $\begin{array}{c}0 \\
100 \\
200 \\
300 \\
\end{array}$ & $\begin{array}{l}0.737 \mathrm{i}-\mathrm{I} \\
0.895 \mathrm{~d}-\mathrm{h} \\
0.942 \mathrm{c}-\mathrm{g} \\
0.886 \mathrm{e}-\mathrm{h} \\
\end{array}$ & $\begin{array}{l}0.721 \mathrm{~h}-\mathrm{k} \\
0.860 \mathrm{e}-\mathrm{j} \\
0.918 \mathrm{c}-\mathrm{h} \\
0.917 \mathrm{c}-\mathrm{h}\end{array}$ & $\begin{array}{l}307.50 \mathrm{f}-\mathrm{i} \\
436.04 \mathrm{~d}-\mathrm{f} \\
324.67 \mathrm{f}-\mathrm{h} \\
331.29 \mathrm{f}-\mathrm{h}\end{array}$ & $\begin{array}{c}255.89 \mathrm{~g}-\mathrm{i} \\
374.33 \text { ef } \\
402.61 \mathrm{ef} \\
345.62 \mathrm{f}\end{array}$ & $\begin{array}{l}0.495 \text { e-h } \\
0.640 \text { c-f } \\
0.697 \text { b-f } \\
0.775 \text { b-e }\end{array}$ & $\begin{array}{l}0.260 \mathrm{e}-\mathrm{g} \\
0.325 \mathrm{e}-\mathrm{g} \\
0.349 \mathrm{~d}-\mathrm{g} \\
0.442 \mathrm{c}-\mathrm{e}\end{array}$ \\
\hline 6000 & $\begin{array}{c}0 \\
100 \\
200 \\
300\end{array}$ & $\begin{array}{c}0.729 \mathrm{j}-\mathrm{m} \\
0.860 \mathrm{e}-\mathrm{j} \\
0.883 \mathrm{e}-\mathrm{i} \\
0.841 \mathrm{f}-\mathrm{I}\end{array}$ & $\begin{array}{c}0.714 \mathrm{~h}-\mathrm{k} \\
0.845 \mathrm{e}-\mathrm{j} \\
0.902 \mathrm{~d}-\mathrm{i} \\
0.828 \mathrm{f}-\mathrm{j}\end{array}$ & $\begin{array}{c}429.17 \text { ef } \\
612.58 \mathrm{a}-\mathrm{c} \\
566.15 \mathrm{~b}-\mathrm{d} \\
506.06 \mathrm{c}-\mathrm{e}\end{array}$ & $\begin{array}{l}388.42 \text { ef } \\
562.70 \mathrm{ab} \\
562.33 \mathrm{ab} \\
476.20 \mathrm{~cd}\end{array}$ & $\begin{array}{l}0.432 \mathrm{f}-\mathrm{h} \\
0.607 \mathrm{c}-\mathrm{g} \\
0.737 \mathrm{~b}-\mathrm{f} \\
0.661 \mathrm{c}-\mathrm{f}\end{array}$ & $\begin{array}{c}0.233 \mathrm{fg} \\
0.313 \mathrm{e}-\mathrm{g} \\
0.253 \mathrm{e}-\mathrm{g} \\
0.274 \mathrm{e}-\mathrm{g}\end{array}$ \\
\hline 8000 & $\begin{array}{c}0 \\
100 \\
200 \\
300 \\
\end{array}$ & $\begin{array}{l}0.704 \mathrm{Im} \\
0.778 \mathrm{~h}-\mathrm{I} \\
0.854 \mathrm{f}-\mathrm{k} \\
0.793 \mathrm{~h}-\mathrm{I} \\
\end{array}$ & $\begin{array}{c}0.689 \mathrm{i}-\mathrm{k} \\
0.788 \mathrm{f}-\mathrm{j} \\
0.887 \mathrm{e}-\mathrm{j} \\
0.773 \mathrm{~g}-\mathrm{k}\end{array}$ & $\begin{array}{c}427.33 \text { ef } \\
666.53 \mathrm{ab} \\
746.35 \mathrm{a} \\
718.70 \mathrm{a} \\
\end{array}$ & $\begin{array}{c}432.33 \mathrm{de} \\
546.65 \mathrm{ab} \\
576.67 \mathrm{a} \\
583.67 \mathrm{a}\end{array}$ & $\begin{array}{c}0.289 \mathrm{~h} \\
0.701 \mathrm{~b}-\mathrm{f} \\
0.644 \mathrm{c}-\mathrm{f} \\
0.472 \mathrm{e}-\mathrm{h}\end{array}$ & $\begin{array}{l}0.188 \mathrm{fg} \\
0.243 \mathrm{f}-\mathrm{g} \\
0.238 \mathrm{fg} \\
0.199 \mathrm{fg} \\
\end{array}$ \\
\hline 10000 & $\begin{array}{c}0 \\
100 \\
200 \\
300\end{array}$ & $\begin{array}{c}0.597 \mathrm{~m} \\
0.749 \mathrm{~h}-\mathrm{I} \\
0.790 \mathrm{~h}-\mathrm{I} \\
0.710 \mathrm{k}-\mathrm{m}\end{array}$ & $\begin{array}{c}0.566 \mathrm{k} \\
0.765 \mathrm{~h}-\mathrm{k} \\
0.673 \mathrm{jk} \\
0.697 \mathrm{~h}-\mathrm{k}\end{array}$ & $\begin{array}{c}401.72 \mathrm{e}-\mathrm{g} \\
737.73 \mathrm{a} \\
628.76 \mathrm{a}-\mathrm{c} \\
566.20 \mathrm{~b}-\mathrm{d}\end{array}$ & $\begin{array}{c}499.67 \mathrm{bc} \\
560.77 \mathrm{ab} \\
591.00 \mathrm{a} \\
530.75 \mathrm{a}-\mathrm{c}\end{array}$ & $\begin{array}{c}0.227 \mathrm{~h} \\
0.341 \mathrm{gh} \\
0.284 \mathrm{~h} \\
0.325 \mathrm{gh}\end{array}$ & $\begin{array}{l}0.152 \mathrm{~g} \\
0.227 \mathrm{fg} \\
0.200 \mathrm{fg} \\
0.189 \mathrm{fg}\end{array}$ \\
\hline
\end{tabular}

Means within a column in the same group followed by the same letter are not significantly different $(P=0.05)$ according to Duncan's multiple range test 
Table 6. Effect of irrigation by saline water and foliar spraying of salicylic acid on nitrogen, phosphorus, potassium and calcium percentages of Calendula officinalis L. plant in 2014/2015 and 2015/2016 seasons

\begin{tabular}{|c|c|c|c|c|c|c|c|c|c|}
\hline \multirow{2}{*}{\multicolumn{2}{|c|}{ Treatments }} & \multicolumn{2}{|c|}{$\begin{array}{c}\text { Nitrogen } \\
\text { percentage }\end{array}$} & \multicolumn{2}{|c|}{$\begin{array}{l}\text { Phosphorus } \\
\text { percentage }\end{array}$} & \multicolumn{2}{|c|}{$\begin{array}{l}\text { Potassium } \\
\text { percentage }\end{array}$} & \multicolumn{2}{|c|}{$\begin{array}{l}\text { Calcium } \\
\text { percentage }\end{array}$} \\
\hline & & $\begin{array}{c}1^{\text {st }} \\
\text { Season }\end{array}$ & $\begin{array}{c}2^{\text {nd }} \\
\text { Season }\end{array}$ & $\begin{array}{c}1^{\text {st }} \\
\text { Season }\end{array}$ & $\begin{array}{c}2^{\text {nd }} \\
\text { Season }\end{array}$ & $\begin{array}{c}1^{\text {st }} \\
\text { Season }\end{array}$ & $\begin{array}{c}2^{\text {nd }} \\
\text { Season }\end{array}$ & $\begin{array}{c}1^{\text {st }} \\
\text { Season }\end{array}$ & $\begin{array}{c}2^{\text {nd }} \\
\text { Season }\end{array}$ \\
\hline \multicolumn{10}{|c|}{ Salinity level (ppm) } \\
\hline \multicolumn{2}{|c|}{ Control(300) } & $1.689 \mathrm{a}$ & $1.889 \mathrm{a}$ & $0.195 \mathrm{a}$ & $0.185 \mathrm{a}$ & $1.794 \mathrm{a}$ & $1.703 \mathrm{a}$ & $1.861 \mathrm{f}$ & $1.889 \mathrm{~d}$ \\
\hline \multicolumn{2}{|c|}{2000} & $1.434 \mathrm{~b}$ & $1.623 \mathrm{~b}$ & $0.179 b$ & $0.167 \mathrm{~b}$ & $1.583 \mathrm{~b}$ & $1.423 b$ & $2.006 \mathrm{e}$ & $2.022 \mathrm{c}$ \\
\hline \multicolumn{2}{|c|}{4000} & $1.351 \mathrm{c}$ & $1.492 \mathrm{c}$ & $0.166 \mathrm{c}$ & $0.141 \mathrm{c}$ & $1.414 \mathrm{c}$ & $1.269 \mathrm{c}$ & $2.072 \mathrm{~d}$ & $2.056 \mathrm{c}$ \\
\hline \multicolumn{2}{|c|}{6000} & $1.223 \mathrm{~d}$ & $1.362 \mathrm{~d}$ & $0.147 d$ & $0.124 d$ & $1.117 d$ & $1.100 \mathrm{~d}$ & $2.122 \mathrm{c}$ & $2.078 \mathrm{c}$ \\
\hline \multicolumn{2}{|c|}{8000} & $1.105 \mathrm{e}$ & $1.168 \mathrm{e}$ & $0.129 \mathrm{e}$ & $0.111 \mathrm{e}$ & $1.106 \mathrm{~d}$ & 0.943 e & $2.200 \mathrm{~b}$ & $2.183 b$ \\
\hline \multicolumn{2}{|c|}{10000} & $1.038 \mathrm{e}$ & $1.022 \mathrm{f}$ & $0.106 \mathrm{f}$ & $0.085 f$ & $0.889 \mathrm{e}$ & $0.800 \mathrm{f}$ & $2.294 \mathrm{a}$ & $2.289 \mathrm{a}$ \\
\hline \multicolumn{10}{|c|}{ SA concentration (ppm) } \\
\hline \multicolumn{2}{|c|}{ Control (0) } & $1.192 \mathrm{c}$ & $1.250 \mathrm{~b}$ & $0.131 \mathrm{c}$ & $0.104 \mathrm{c}$ & $1.208 \mathrm{~b}$ & $1.088 b$ & $2.026 \mathrm{c}$ & $2.019 \mathrm{c}$ \\
\hline \multicolumn{2}{|c|}{100} & $1.413 \mathrm{a}$ & $1.504 \mathrm{a}$ & $0.163 \mathrm{a}$ & $0.151 \mathrm{a}$ & $1.366 \mathrm{a}$ & $1.263 \mathrm{a}$ & $2.137 \mathrm{a}$ & $2.119 a b$ \\
\hline \multicolumn{2}{|c|}{200} & $1.358 \mathrm{a}$ & $1.484 \mathrm{a}$ & $0.167 \mathrm{a}$ & $0.150 \mathrm{a}$ & $1.350 \mathrm{a}$ & $1.265 \mathrm{a}$ & $2.141 \mathrm{a}$ & $2.148 \mathrm{a}$ \\
\hline \multicolumn{2}{|c|}{300} & $1.263 \mathrm{~b}$ & $1.467 \mathrm{a}$ & $0.154 b$ & $0.137 \mathrm{~b}$ & $1.345 \mathrm{a}$ & $1.210 \mathrm{a}$ & $2.067 \mathrm{~b}$ & $2.059 \mathrm{bc}$ \\
\hline \multicolumn{10}{|c|}{ Salinity * SA } \\
\hline \multirow{4}{*}{ Control } & 0 & $1.540 \mathrm{~cd}$ & $1.770 \mathrm{~b}$ & $0.189 \mathrm{a}-\mathrm{c}$ & $0.181 \mathrm{a}-\mathrm{c}$ & $1.600 \mathrm{a}-\mathrm{d}$ & $1.623 \mathrm{a}-\mathrm{c}$ & $1.756 \mathrm{k}$ & $1.822 \mathrm{~g}$ \\
\hline & 100 & $1.881 \mathrm{a}$ & $1.967 \mathrm{a}$ & 0.199 a & $0.190 \mathrm{a}$ & $1.886 a b$ & $1.669 a b$ & $1.978 \mathrm{~h}-\mathrm{j}$ & $1.933 \mathrm{e}-\mathrm{g}$ \\
\hline & 200 & $1.727 \mathrm{ab}$ & $1.897 \mathrm{a}$ & $0.198 a b$ & $0.184 a b$ & $1.931 \mathrm{a}$ & $1.794 \mathrm{a}$ & 1.911 j & $1.956 \mathrm{~d}-\mathrm{g}$ \\
\hline & 300 & $1.610 \mathrm{bc}$ & $1.923 \mathrm{a}$ & $0.197 \mathrm{ab}$ & $0.183 a b$ & $1.760 \mathrm{ab}$ & $1.726 \mathrm{a}$ & $1.800 \mathrm{k}$ & $1.844 \mathrm{fg}$ \\
\hline \multirow{4}{*}{2000} & 0 & $1.274 \mathrm{f}-\mathrm{h}$ & $1.471 \mathrm{fg}$ & $0.163 \mathrm{~d}-\mathrm{f}$ & $0.152 d-f$ & $1.406 \mathrm{c}-\mathrm{f}$ & $1.314 \mathrm{~d}-\mathrm{h}$ & $1.978 \mathrm{~h}-\mathrm{j}$ & $1.933 \mathrm{e}-\mathrm{g}$ \\
\hline & 100 & $1.610 \mathrm{bc}$ & $1.727 \mathrm{bc}$ & $0.184 a-c$ & $0.178 \mathrm{a}-\mathrm{c}$ & $1.669 \mathrm{a}-\mathrm{c}$ & $1.463 \mathrm{c}-\mathrm{e}$ & $2.067 \mathrm{f}-\mathrm{h}$ & $2.044 \mathrm{~b}-\mathrm{g}$ \\
\hline & 200 & $1.465 \mathrm{c}-\mathrm{e}$ & $1.654 \mathrm{~cd}$ & $0.188 a-c$ & $0.171 \mathrm{a}-\mathrm{d}$ & $1.623 \mathrm{a}-\mathrm{d}$ & $1.520 \mathrm{~b}-\mathrm{d}$ & $2.044 \mathrm{~g}-\mathrm{i}$ & $2.111 \mathrm{~b}-\mathrm{e}$ \\
\hline & 300 & $1.386 \mathrm{~d}-\mathrm{f}$ & $1.638 \mathrm{ce}$ & $0.182 \mathrm{a}-\mathrm{d}$ & $0.168 \mathrm{~b}-\mathrm{d}$ & $1.634 \mathrm{a}-\mathrm{c}$ & $1.394 \mathrm{~d}-\mathrm{f}$ & $1.933 \mathrm{ij}$ & $2.000 \mathrm{c}-\mathrm{g}$ \\
\hline \multirow{4}{*}{4000} & 0 & $1.237 \mathrm{f}-\mathrm{h}$ & $1.309 \mathrm{~h}$ & $0.140 \mathrm{~g}$ & $0.089 \mathrm{j}$ & $1.291 \mathrm{~d}-\mathrm{h}$ & $1.166 \mathrm{~g}-\mathrm{j}$ & $2.044 \mathrm{~g}-\mathrm{i}$ & $2.067 b-f$ \\
\hline & 100 & $1.563 \mathrm{bc}$ & $1.570 \mathrm{~d}-\mathrm{f}$ & $0.175 \mathrm{c}-\mathrm{e}$ & $0.162 \mathrm{c}-\mathrm{e}$ & $1.406 \mathrm{c}-\mathrm{f}$ & $1.314 \mathrm{~d}-\mathrm{h}$ & $2.089 \mathrm{f}-\mathrm{h}$ & $2.111 \mathrm{~b}-\mathrm{e}$ \\
\hline & 200 & $1.344 \mathrm{e}-\mathrm{g}$ & 1.548 ef & $0.178 \mathrm{~b}-\mathrm{d}$ & $0.158 d-f$ & $1.383 \mathrm{c}-\mathrm{g}$ & $1.349 \mathrm{~d}-\mathrm{g}$ & $2.067 f-h$ & $2.000 \mathrm{c}-\mathrm{g}$ \\
\hline & 300 & $1.260 \mathrm{f}-\mathrm{h}$ & 1.542 ef & $0.170 \mathrm{c}-\mathrm{e}$ & $0.155 d-f$ & $1.577 \mathrm{~b}-\mathrm{e}$ & $1.246 \mathrm{f}-\mathrm{i}$ & $2.089 \mathrm{f}-\mathrm{h}$ & $2.044 \mathrm{~b}-\mathrm{g}$ \\
\hline \multirow{4}{*}{6000} & 0 & $1.120 \mathrm{~h}-\mathrm{j}$ & $1.120 \mathrm{jk}$ & $0.111 \mathrm{hi}$ & $0.071 \mathrm{k}$ & $1.074 \mathrm{f}-\mathrm{j}$ & $0.891 \mathrm{k}-\mathrm{m}$ & $2.022 \mathrm{~g}-\mathrm{j}$ & $2.000 \mathrm{c}-\mathrm{g}$ \\
\hline & 100 & $1.255 \mathrm{f}-\mathrm{h}$ & $1.428 \mathrm{~g}$ & $0.163 d-f$ & $0.146 \mathrm{e}-\mathrm{g}$ & $1.246 \mathrm{e}-\mathrm{i}$ & $1.280 \mathrm{e}-\mathrm{h}$ & $2.133 \mathrm{~d}-\mathrm{g}$ & $2.067 b-f$ \\
\hline & 200 & $1.316 \mathrm{e}-\mathrm{g}$ & $1.469 \mathrm{fg}$ & 0.173 c-e & $0.156 \mathrm{~d}-f$ & $1.166 \mathrm{f}-\mathrm{j}$ & $1.120 \mathrm{~h}-\mathrm{j}$ & $2.222 \mathrm{~b}-\mathrm{e}$ & $2.178 \mathrm{a}-\mathrm{d}$ \\
\hline & 300 & $1.199 \mathrm{~g}-\mathrm{i}$ & $1.430 \mathrm{~g}$ & $0.142 \mathrm{~g}$ & $0.122 \mathrm{hi}$ & $0.983 \mathrm{~h}-\mathrm{j}$ & $1.109 \mathrm{~h}-\mathrm{j}$ & $2.111 \mathrm{e}-\mathrm{g}$ & $2.067 b-f$ \\
\hline \multirow{4}{*}{8000} & 0 & $1.059 \mathrm{i}-\mathrm{k}$ & 0.987 I & $0.093 \mathrm{ij}$ & $0.068 \mathrm{k}$ & $1.051 \mathrm{~g}-\mathrm{j}$ & $0.789 \mathrm{Im}$ & $2.133 \mathrm{~d}-\mathrm{g}$ & $2.111 \mathrm{~b}-\mathrm{e}$ \\
\hline & 100 & $1.050 \mathrm{i}-\mathrm{k}$ & $1.242 \mathrm{hi}$ & $0.145 \mathrm{fg}$ & $0.131 \mathrm{gh}$ & $1.086 \mathrm{f}-\mathrm{j}$ & $1.051 \mathrm{i}-\mathrm{k}$ & 2.244 a-d & $2.200 \mathrm{a}-\mathrm{c}$ \\
\hline & 200 & $1.199 \mathrm{~g}-i$ & $1.254 \mathrm{hi}$ & $0.157 \mathrm{e}-\mathrm{g}$ & $0.141 \mathrm{f}-\mathrm{h}$ & $1.074 \mathrm{f}-\mathrm{j}$ & $0.971 \mathrm{j}-\mathrm{I}$ & 2.244 a-d & $2.267 \mathrm{ab}$ \\
\hline & 300 & $1.111 \mathrm{~h}-\mathrm{j}$ & $1.189 \mathrm{ij}$ & $0.121 \mathrm{~h}$ & $0.105 \mathrm{ij}$ & $1.211 \mathrm{f}-\mathrm{i}$ & $0.960 \mathrm{j}-1$ & $2.178 c-f$ & $2.156 \mathrm{a}-\mathrm{e}$ \\
\hline \multirow{4}{*}{10000} & 0 & $0.924 \mathrm{k}$ & $0.840 \mathrm{~m}$ & $0.090 \mathrm{j}$ & $0.063 \mathrm{k}$ & $0.823 \mathrm{j}$ & $0.743 \mathrm{~m}$ & $2.222 b-e$ & $2.178 \mathrm{a}-\mathrm{d}$ \\
\hline & 100 & $1.120 \mathrm{~h}-\mathrm{j}$ & $1.089 \mathrm{j}$ & $0.114 \mathrm{~h}$ & $0.097 \mathrm{j}$ & $0.903 \mathrm{ij}$ & $0.800 \mathrm{~lm}$ & $2.311 a b$ & $2.356 \mathrm{a}$ \\
\hline & 200 & $1.097 \mathrm{~h}-\mathrm{j}$ & $1.079 \mathrm{kl}$ & $0.108 \mathrm{~h}-\mathrm{j}$ & $0.091 \mathrm{j}$ & $0.926 \mathrm{ij}$ & $0.834 \mathrm{~lm}$ & $2.356 \mathrm{a}$ & $2.378 \mathrm{a}$ \\
\hline & 300 & $1.013 \mathrm{jk}$ & $1.081 \mathrm{kl}$ & $0.110 \mathrm{hi}$ & $0.090 \mathrm{j}$ & $0.903 \mathrm{ij}$ & $0.823 \mathrm{Im}$ & $2.289 a-c$ & $2.244 a b$ \\
\hline
\end{tabular}

Means within a column in the same group followed by the same letter are not significantly different $(P=0.05)$ according to Duncan's multiple range test 
Effect of Saline Water Irrigation and Foliar Spraying of Salicylic Acid on Growth, Flowering and Chemical Composition of Pot Marigold (Calendula officinalis L.) Plant

Table 7. Effect of irrigation by saline water and foliar spraying of salicylic acid on sodium, chloride and survival percentages of Calendula officinalis L. plant in 2014/2015 and 2015/2016 seasons

\begin{tabular}{|c|c|c|c|c|c|c|c|}
\hline & & \multicolumn{2}{|c|}{ Sodium Percentage } & \multicolumn{2}{|c|}{ Chloride percentage } & \multicolumn{2}{|c|}{ Survival percentage } \\
\hline \multicolumn{2}{|c|}{ Treatments } & $\begin{array}{c}1^{\text {st }} \\
\text { Season }\end{array}$ & $\begin{array}{c}\text { 2nd } \\
\text { Season }\end{array}$ & $\begin{array}{c}1^{\text {st }} \\
\text { Season }\end{array}$ & $\begin{array}{c}\text { 2nd } \\
\text { Season }\end{array}$ & $\begin{array}{c}1^{\text {st }} \\
\text { Season }\end{array}$ & $\begin{array}{c}\text { 2nd } \\
\text { Season }\end{array}$ \\
\hline \multicolumn{8}{|c|}{ Salinity level (ppm) } \\
\hline \multicolumn{2}{|c|}{ Control(300) } & $0.505 \mathrm{f}$ & $0.586 \mathrm{f}$ & $0.237 \mathrm{f}$ & $0.331 \mathrm{f}$ & $100.00 \mathrm{a}$ & $100.00 \mathrm{a}$ \\
\hline \multicolumn{2}{|c|}{2000} & $0.638 \mathrm{e}$ & $0.842 \mathrm{e}$ & $0.431 \mathrm{e}$ & $0.530 \mathrm{e}$ & $95.00 \mathrm{a}$ & $98.33 \mathrm{a}$ \\
\hline \multicolumn{2}{|c|}{4000} & $0.826 d$ & $0.986 \mathrm{~d}$ & $0.757 d$ & $0.738 d$ & $85.00 \mathrm{~b}$ & $93.33 \mathrm{a}$ \\
\hline \multicolumn{2}{|c|}{6000} & $0.989 \mathrm{c}$ & $1.076 \mathrm{c}$ & $0.890 \mathrm{c}$ & $0.928 \mathrm{c}$ & $68.33 \mathrm{c}$ & $75.00 \mathrm{~b}$ \\
\hline \multicolumn{2}{|c|}{8000} & $1.130 \mathrm{~b}$ & $1.173 b$ & $1.098 b$ & $1.136 \mathrm{~b}$ & $51.67 \mathrm{~d}$ & $55.00 \mathrm{c}$ \\
\hline \multicolumn{2}{|c|}{10000} & $1.314 \mathrm{a}$ & $1.294 \mathrm{a}$ & $1.198 \mathrm{a}$ & $1.188 \mathrm{a}$ & $35.00 \mathrm{e}$ & $41.67 \mathrm{~d}$ \\
\hline \multicolumn{8}{|c|}{ SA concentration (ppm) } \\
\hline & $0.973 \mathrm{a}$ & $1.074 \mathrm{a}$ & $0.839 \mathrm{a}$ & $0.871 \mathrm{a}$ & $66.67 \mathrm{~b}$ & $70.00 \mathrm{c}$ \\
\hline \multicolumn{2}{|c|}{100} & $0.864 \mathrm{~b}$ & $0.954 \mathrm{c}$ & $0.754 \mathrm{~b}$ & $0.805 b$ & $76.67 \mathrm{a}$ & $81.11 a b$ \\
\hline \multicolumn{2}{|c|}{200} & $0.881 \mathrm{~b}$ & $0.941 \mathrm{c}$ & $0.723 \mathrm{c}$ & $0.783 b$ & $76.67 \mathrm{a}$ & $82.22 \mathrm{a}$ \\
\hline \multicolumn{2}{|c|}{300} & $0.884 \mathrm{~b}$ & $1.002 \mathrm{~b}$ & $0.757 \mathrm{~b}$ & $0.776 \mathrm{~b}$ & $70.00 \mathrm{~b}$ & $75.56 \mathrm{bc}$ \\
\hline \multicolumn{8}{|c|}{ Salinity * SA } \\
\hline \multirow{4}{*}{ Control } & 0 & $0.544 \mathrm{j}-\mathrm{I}$ & $0.626 \mathrm{i}$ & $0.265 I$ & $0.360 \mathrm{k}$ & $100.00 \mathrm{a}$ & $100.00 \mathrm{a}$ \\
\hline & 100 & $0.524 \mathrm{kl}$ & $0.551 \mathrm{i}$ & $0.227 \mid$ & $0.303 \mathrm{k}$ & $100.00 \mathrm{a}$ & $100.00 \mathrm{a}$ \\
\hline & 200 & 0.4521 & $0.566 \mathrm{i}$ & $0.208 \mid$ & $0.341 \mathrm{k}$ & $100.00 \mathrm{a}$ & $100.00 \mathrm{a}$ \\
\hline & 300 & $0.501 \mathrm{kl}$ & $0.599 \mathrm{i}$ & 0.246 I & $0.322 \mathrm{k}$ & $100.00 \mathrm{a}$ & $100.00 \mathrm{a}$ \\
\hline \multirow{4}{*}{2000} & 0 & $0.669 \mathrm{i}$ & $0.796 \mathrm{~h}$ & $0.492 \mathrm{j}$ & $0.549 \mathrm{j}$ & $86.67 \mathrm{a}-\mathrm{c}$ & $93.33 \mathrm{a}$ \\
\hline & 100 & 0.576 jk & $0.896 \mathrm{gh}$ & $0.417 \mathrm{jk}$ & $0.568 \mathrm{ij}$ & $100.00 \mathrm{a}$ & $100.00 \mathrm{a}$ \\
\hline & 200 & 0.684 i & $0.848 \mathrm{~h}$ & $0.360 \mathrm{k}$ & $0.492 \mathrm{j}$ & $100.00 \mathrm{a}$ & $100.00 \mathrm{a}$ \\
\hline & 300 & $0.624 \mathrm{ij}$ & $0.828 \mathrm{~h}$ & $0.454 \mathrm{j}$ & $0.511 \mathrm{j}$ & $93.33 \mathrm{ab}$ & $100.00 \mathrm{a}$ \\
\hline \multirow{4}{*}{4000} & 0 & $0.891 \mathrm{fg}$ & $1.090 \mathrm{de}$ & $0.909 \mathrm{fg}$ & $0.757 \mathrm{~g}$ & $80.00 \mathrm{~b}-\mathrm{d}$ & $86.67 \mathrm{a}$ \\
\hline & 100 & $0.798 \mathrm{~h}$ & $0.978 \mathrm{fg}$ & $0.795 \mathrm{~h}$ & $0.852 \mathrm{f}$ & $86.67 \mathrm{a}-\mathrm{c}$ & $100.00 \mathrm{a}$ \\
\hline & 200 & $0.776 \mathrm{~h}$ & $0.866 \mathrm{~h}$ & 0.644 i & $0.701 \mathrm{gh}$ & $93.33 \mathrm{ab}$ & $93.33 \mathrm{a}$ \\
\hline & 300 & $0.841 \mathrm{gh}$ & 1.008 ef & $0.682 \mathrm{i}$ & $0.644 \mathrm{hi}$ & $80.00 \mathrm{~b}-\mathrm{d}$ & $93.33 \mathrm{a}$ \\
\hline \multirow{4}{*}{6000} & 0 & $1.083 \mathrm{~cd}$ & $1.245 \mathrm{bc}$ & $0.966 \mathrm{f}$ & $1.060 \mathrm{e}$ & $66.67 \mathrm{~d}-\mathrm{f}$ & $60.00 \mathrm{bc}$ \\
\hline & 100 & 0.936 ef & 1.013 ef & $0.852 \mathrm{gh}$ & $0.890 \mathrm{f}$ & 73.33 c-e & $86.67 \mathrm{a}$ \\
\hline & 200 & $0.996 \mathrm{de}$ & $0.981 \mathrm{fg}$ & $0.871 \mathrm{gh}$ & $0.890 \mathrm{f}$ & $73.33 \mathrm{c}-\mathrm{e}$ & $86.67 \mathrm{a}$ \\
\hline & 300 & 0.941 ef & 1.065 ef & $0.871 \mathrm{gh}$ & $0.871 \mathrm{f}$ & 60.00 ef & $66.67 \mathrm{~b}$ \\
\hline \multirow{4}{*}{8000} & 0 & $1.267 \mathrm{~b}$ & $1.290 \mathrm{bc}$ & $1.155 b-d$ & $1.212 \mathrm{ab}$ & $40.00 \mathrm{gh}$ & $46.67 \mathrm{c}-\mathrm{e}$ \\
\hline & 100 & $1.078 \mathrm{~cd}$ & $1.100 \mathrm{de}$ & $1.060 \mathrm{e}$ & $1.079 \mathrm{de}$ & 60.00 ef & $53.33 \mathrm{~b}-\mathrm{d}$ \\
\hline & 200 & $1.083 \mathrm{~cd}$ & $1.108 \mathrm{de}$ & $1.098 \mathrm{c}-\mathrm{e}$ & $1.117 \mathrm{c}-\mathrm{e}$ & $53.33 \mathrm{fg}$ & $66.67 \mathrm{~b}$ \\
\hline & 300 & $1.093 \mathrm{c}$ & $1.193 \mathrm{~cd}$ & $1.079 \mathrm{de}$ & $1.136 \mathrm{~b}-\mathrm{e}$ & $53.33 \mathrm{fg}$ & $53.33 \mathrm{~b}-\mathrm{d}$ \\
\hline \multirow{4}{*}{10000} & 0 & $1.385 \mathrm{a}$ & $1.397 \mathrm{a}$ & $1.250 \mathrm{a}$ & $1.287 \mathrm{a}$ & $26.67 \mathrm{~h}$ & $33.33 \mathrm{e}$ \\
\hline & 100 & $1.270 \mathrm{~b}$ & $1.185 \mathrm{~cd}$ & $1.174 \mathrm{a}-\mathrm{c}$ & $1.136 \mathrm{~b}-\mathrm{e}$ & $40.00 \mathrm{gh}$ & $46.67 \mathrm{c}-\mathrm{e}$ \\
\hline & 200 & $1.297 \mathrm{ab}$ & $1.275 \mathrm{bc}$ & $1.155 \mathrm{~b}-\mathrm{d}$ & $1.155 \mathrm{~b}-\mathrm{d}$ & $40.00 \mathrm{gh}$ & $46.67 \mathrm{c}-\mathrm{e}$ \\
\hline & 300 & $1.305 \mathrm{ab}$ & $1.320 \mathrm{ab}$ & $1.212 \mathrm{ab}$ & $1.174 \mathrm{bc}$ & $33.33 \mathrm{~h}$ & $40.00 \mathrm{de}$ \\
\hline
\end{tabular}

Means within a column in the same group followed by the same letter are not significantly different $(P=0.05)$ according to Duncan's multiple range test

\section{DISCUSSION}

The SA spraying applications increased the vegetative and roots growth characters, these increments may be attributed to the increment in leaf total chlorophyll as found in Table (5) or the increment of the plant nutrients, i.e. nitrogen, phosphorus and potassium percentages in the vegetative growth as mentioned in Table (6). These nutrients play roles in the development of the plant; nitrogen 
increase the vegetative growth of the plant, phosphorus play role in roots formation and flowering of the plants, while potassium play role in the translocation of carbohydrates in the plant. Also, salicylic acid has been found to play a key role in the regulation of plant growth, development and in the responses to environmental stresses (Raskin, 1992 a, b, Popova et al 1997, Senaratna et al 2000 and Shakirova et al 2003). The stimulating effect of SA on the plant growth may be attributed to the influence of SA on a range of diverse processes in plants, including stomatal closure, ion uptake and transport (Harper and Balke, 1981, Khan et al 2003, Gunes et al 2005 and Aftab et al 2010), membrane permeability (Barkosky and Einhellig, 1993), photosynthetic and growth rates (Khan et al 2003), as well as nitrogen metabolism, proline metabolism, production of glycinebetaine, antioxidant defense system, and plant water relations under stress conditions and thereby provides protection in plants against abiotic stresses (Khan et al 2003, Nazar et al 2011 and Miura \& Tada, 2014). In addition to facilitating plant growth, $S A$ has been shown as an important signal molecule which can induce particular enzyme catalyzing biosynthetic reactions.

Concerning the flowering parameters, SA spraying applications increased the flowering parameters. These increments in the flowering parameters may be considered as a result to the increment in the vegetative growth characters, i.e. plant height, number of leaves/plant, leaf area, fresh and dry weights of the vegetative growth and roots as found in Tables (1, 2 and 3 ) or may be attributed to the increase in leaf total chlorophyll as found in Table (5) or the increment in plant nutrients as mentioned in Table (6), i.e. nitrogen, phosphorus and potassium percentages in vegetative growth. Moreover, SA has been found that it promotes flowering, enhances flowers longevity, inhibits ethylene biosynthesis and reverses the ABA impacts (Raskin et al 1992a and Martinez et al 2004).

Concerning the effect of saline water irrigation on plant growth and chlorophyll content, salinity leaded to decrease chlorophyll content that is because of the plant digress toward producing nitrogen compound such as proline. While, SA increased photosynthesis under salt stress by decreasing cellular $\mathrm{Na}^{+}$and $\mathrm{Cl}^{-}$ions and increasing the content of nutrients (Gunes et al 2007). Under salinity stress, an increase in $\mathrm{NaCl}$ in plants leads to an increase in sodium and chloride ions but may result a decrease in the other nutrients such as $\mathrm{N}$,
$\mathrm{P}, \mathrm{K}$ and $\mathrm{Ca}$. Also, salinity affects the nutritional balance of $\mathrm{NaCl}$ result in higher levels of $\mathrm{Na}^{+} / \mathrm{Ca}^{2+}$, $\mathrm{Na}^{+} / \mathrm{K}^{+}, \mathrm{Na}^{+} / \mathrm{Mg}^{2+}, \mathrm{Cl}^{-} / \mathrm{NO}_{3}{ }^{-}$and $\mathrm{Cl}^{-} / \mathrm{H}_{2} \mathrm{PO}_{4}{ }^{-}$, thus causing plant growth retardation, Sodium and chloride can influence the uptake of nutrients by competing with nutrients or affecting the ion permeability of membrane (Grattan and Grieve 1999).

\section{CONCLUSION}

This study demonstrated that foliar spraying of SA under saline water irrigation conditions induced positive effects on the plant growth, flowering parameters, and the contents of chlorophyll, flowercarotene, proline and minerals of vegetative growth of pot marigold plants. Moreover in most cases, under the same saline water irrigation conditions spraying SA at 100 and 200 ppm concentrations alleviated the salinity effect on the plants, enhanced the plant growth and increased the flowering parameters. The most effective treatments which enhance growth, flowering parameters, chlorophyll content, carotene contents in flowers, proline and mineral contents were found to be 100 and 200 ppm SA sprayings under nonsaline conditions. Further studies are required in order to determine the effect of SA under saline conditions on the net photosynthetic rate, water relations, antioxidant compounds, enzyme activity and endogenous phytohormones.

\section{REFERENCES}

Aftab, T., Khan, M.M.A., Idrees, M., Naeem, M. and Moinuddin 2010. Salicylic acid acts as potent enhancer of growth, photosynthesis and artemisinin production in Artemisia annua L.. J. Crop Sci. Biotech., 13(3), 183-188.

Alarcon, J.J., Sanchez-Blanco, M.J., Bolarin, M.C. and Torrecillas, A. 1994. Growth and osmotic adjustment of two tomato cultivars during and after saline stress. Plant and Soil, 166, 75-82.

Allakhverdiev, S.I., Sakamoto, A., Nishiyama, Y., Inaba, M. and Murata, N. 2000. Ionic and osmotic effects of $\mathrm{NaCl}$-induced inactivation of photosystems I and II in Synechococcus sp.. Plant Physiol., 123(3), 1047-1056.

AOAC, 2005. Association of Official Analytical Chemists-International, Official Methods of Analysis, $18^{\text {th }}$, Horwitz W. and Latimer G.W. (eds.), 107 p., AOAC-Int. Suite 500, 481 North Frederick Avenue, Gaithersburg, Maryland, USA. 
Effect of Saline Water Irrigation and Foliar Spraying of Salicylic Acid on Growth, Flowering and Chemical Composition of Pot Marigold (Calendula officinalis L.) Plant

Bandurska, H. and Stroiński, A. 2005. The effect of salicylic acid on barley response to water deficit. Acta Physioligae Plantarum, 27(3), 379-386.

Barkosky, R.R. and Einhellig, F.A. 1993. Effects of salicylic acid on plant water relationship. $\mathbf{J}$. Chem. Ecol., 19(2), 237-247.

Bates, L.S., Waldren, R.P. and Teare, I.D. 1973. Rapid determination of free proline for water stress studies. Plant and soil, 39, 205-207.

Bayat, H., Alirezaie, M. and Neamati, H. 2012. Impact of exogenous salicylic acid on growth and ornamental characteristics of calendula (Calendula officinalis L.) under salinity stress. J. stress Physiol. \& Biochem., 8(1), 258-267.

Brown, J.D. and Lilleland, O. 1946. Rapid determination of potassium and sodium in plant material and soil extract by flame photometery. Proc. Amer. Soc. Hort. Sci., 48, 341-346.

Carter, C.T., Grieve, C.M., Poss, J.A. and Suarez, D.L. 2005. Production and ion uptake of Celosia argenta irrigated with saline wastewaters. Scientia Horticulturae, 106(3), 381-394.

Chartzoulakis, K., Loupassaki, M., Bertaki, M. and Androulakis, I. 2002. Effects of $\mathrm{NaCl}$ salinity on growth, ion content and $\mathrm{CO} 2$ assimilation rate of six olive cultivars. Scientia Horticulturae, 96, 235-247.

Cheng, K.L. and Bray, R.H. 1951. Determination of Calcium and Magnesium in Soil and Plants materials. Soil Sci., 72(6), 449-458.

Dole, J.M. and Wilkins, H.F. 2004. Floriculture: Principles and species, (2 ${ }^{\text {nd }}$ Ed.). 226 p., Prentice Hall, Upper Saddle River, New Jersey, USA.

Earle, F.R., Mikolajczak, K.L., Wolff, I.A. and Barclay, A.S. 1964. Search of new industrial oils $X$ Seed oils of the Calenduleae. J. Amer. Oil Chem. Soc., 41(5), 345-347.

Elwan, M.W.M. and El-Hamahmy, M.A.M. 2009. Improved productivity and quality associated with salicylic acid application in greenhouse pepper. Scientia Horticulturae, 122(4), 521526.

Fatemi, R. and Aboutalebi, A. 2012. Evaluation the interaction of salinity and salicylic acid on Sweet basil (ocimum basilicum) properties. Annals Bio. Res., 3(11), 5106-5109.

Fathy, E.L.E, Abd-Rahman, A.M.M. and Khedr, Z.M.A. 2003. Response of broad bean to foliar spray of different K-sources and energy related organic compounds (EROC) to induce better internal $\mathrm{K}$ and sugars case towards better growth and productivity. J. Agric. Sci., Mansoura Univ., 28(4), 2935-2954.

Ghai, N., Setia, R.C. and Setia, N. 2002. Effects of paclobutrazol and salicylic acid on chlorophyll content, hill activity and yield components in Brassica napus L. (cv. GSL-1). Phytomorphol., 52, 83-87.

Grattan, S.R. and Grieve, C.M. 1999. Mineral nutrient acquisition and response by plants grown in saline environments. In: Pessarakli, M. (ed.) Handbook of plant and crop stress, $\left(2^{\text {nd }}\right.$ ed.). pp. 203-229, CRC Press, Boca Raton, Florida, USA.

Gunes, A., Inal, A., Alpaslan, M., Cicek, N., Guneri, E., Eraslan, F. and Guzelordu, T. 2005. Effects of exogenously applied salicylic acid on the induction of multiple stress tolerance and mineral nutrition in maize (Zea mays L.). Arch Agron. Soil Sci., 51(6), 687-695.

Gunes, A., Inal, A., Alpaslan, M., Eraslan, F., Bagci, E.G. and Cicek, N. 2007. Salicylic acid induced changes on some physiological parameters symptomatic for oxidative stress and mineral nutrition in maize (Zea mays L.) grown under salinity. J. Plant Physiol., 164(6), 728736.

Hara, M., Furukawa, J., Sato, A., Mizoguchi, T. and Miura, K. 2012. Abiotic stress and role of salicylic acid in plants. In: Ahmad P. and Prasad M.N.V. (eds.), Abiotic stress responses in plants. pp. 235-251, Springer, New York, USA.

Harper, J.R. and Balke, N.E. 1981. Characterization of the inhibition of $\mathrm{K}^{+}$absorption in oat roots by salicylic acid. Plant Physiol., 68(6), 1349-1353.

Hashish, KH., Mazhar, A., Zaghloul, S., Abdel Aziz, N., Mahgoub, M. and Eid, R. 2015b. Application of salicylic acid on Calendula officinalis $\mathrm{L}$. is to alleviate the adverse effects of salinity stress. Intern. J. Chemtech Res., 8(6), 379-388.

Hashish, KH.L., Mazhar, A., Abdel Aziz, N. and Mahgoub, M. 2015a. The influence of different levels of foliar-application SA on the flowering and some chemical compositions of Calendula officinalis L. under salinity irrigation. Intern. J. Chemtech Res., 8(6), 890-897.

He, Y. and Zhu, Z.J. 2008. Exogenous salicylic acid alleviates $\mathrm{NaCl}$ toxicity and increases antioxidative enzyme activity in Lycopersicon esculentum. Biologia Plantarum, 52(4), 792-795. 
Kandeel, Y.M. 2004. Effect of salinity and growth regulators on growth and chemical compostion of Hibiscus rosasinensis L. transplants. J. Agric. Res. Tanta Univ., 30(4), 944-963.

Karlidag, H., Yildirim, E. and Turan, M. 2009. Salicylic acid ameliorates the adverse effect of salt stress on strawberry. Sci. Agric. Piracicaba, Braz., 66(2), 180-187.

Kaya, C., Higgs, D., Ince, F., Amador, B.M., Cakir, A. and Sakar, E. 2003. Ameliorative effects of potassium phosphate on salt stressed pepper and cucumber. J. of Plant Nutrition. 26(4), 807-820.

Khan, N.A., Syeed, S., Masood, A., Nazar, R. and Iqbal, N. 2010. Application of salicylic acid increases contents of nutrients and antioxidative metabolism in mungbean and alleviates adverse effects of salinity stress. Int. J. Plant Biol. 1(1), 1-8.

Khan, W., Prithiviraj, B. and Smith, D.L. 2003. Photosynthetic responses of corn and soybean to foliar application of salicylates. J. of Plant Physiology, 160(5), 485-492.

Kishimoto, S., Maoka, T., Sumitomo, K. and Ohmiya, A. 2005. Analysis of Carotenoid Composition in Petals of Calendula (Calendula officinalis L.). Biosci. Biotechnol. Biochem. 69(11), 2122-2128.

Kraemer, E.O. and Stamm, A.J. 1924. Mohr's Method for the Determination of Silver and Halogens in other than Neutral Solutions, J. Am. Chem. Soc., 46(12), 2707- 2709.

Kuznetsov, VI.V. and Shevyakova, N.I. 1999. Proline under stress conditions: Biological role, metabolism and regulation. Russian J. of Plant Physiol., 46, 321-336.

Mady, M.A. 2009. Effect of foliar application with salicylic acid and vitamin $E$ on growth and productivity of tomato (Lycopersicon esculentum Mill.) plant. J. Agric. Sci. Mansoura Univ., 34(6), 6735-6746.

Martínez, C., Pons, E., Prats, G. and León, J. 2004. Salicylic acid regulates flowering time and links defence responses and reproductive development. Plant J., 37(2), 209-217.

Miura, K., and Tada, Y. 2014. Regulation of water, salinity, and cold stress responses by salicylic acid. Front. Plant Sci. 5(4),1-12.

Moharekar, S.T., Lokhande, S.D., Hara, T., Tanaka, R., Tanaka, A. and Chavan, P.D. 2003. Effect of salicylic acid on chlorophyll and carotenoid contents of wheat and moong seedlings. Photosynthetica, 41(2), 315-317.
Moursi, M.A., Tawfik, H.A. and Abdel-Gawad, A. 1968. Principles of agricultural researches (in Arabic). 418 p., Dar El-Hanna printing House, Cairo, Egypt.

Nagata, M. and Yamashita, I. 1992. Simple method for simultaneous determination of chlorophyll and carotenoids in tomato fruit. J. Japan. Soc. Food Sci. Technol., 39 (10), 925-928.

Najafian, S., khoshkhui, M. and Tavallali, V. 2009a. Effect of Salicylic Acid and Salinity in Rosemary (Rosmarinus officinalis L.): Investigation on Changes in Gas Exchange, Water Relations, and Membrane Stabilization. Advances in Environmental Biology, 3(3), 322328.

Najafian, S., khoshkhui, M., Tavallali, V. and Saharkhiz, M.J. 2009b. Effect of Salicylic Acid and Salinity in Thyme (Thymus Vulgaris L.): Investigation on Changes in Gas Exchange, Water Relations, and Membrane Stabilization and Biomass Accumulation. Australian J. Basic and Appl. Sci., 3(3), 2620-2626.

Nazar, R., lqbal, N., Syeed, S. and Khan, N.A. 2011. Salicylic acid alleviates decreases in photosynthesis under salt stress by enhancing nitrogen and sulfur assimilation and antioxidant metabolism differentially in two mungbean cultivars. J. Plant Physiol., 168(8), 807-815.

Nofal, F.H., El-Segai, M.U. and Seleem, E.A. 2015. Response of Calendula officinalis $\mathrm{L}$. Plants to Growth Stimulants under Salinity Stress. American-Eurasian J. Agric. \& Environ. Sci., 15(9), 1767-1778.

Pacheco, A.C., Cabral, C., Fermino, E. and Aleman, C. 2013. Salicylic acid-induced changes to growth, flowering and flavonoids production in marigold plants. J. of med. Plants Res., 7(42), 3158-3163.

Page, A.L., Miller, R.H. and Keeney, D.R. 1982. Methods of soil analysis-chemical and microbiology properties, Part 2. 228 p., SSSA Inc., Mad., WI., USA.

Parida, A.K. and Das, A.B. 2005. Salt tolerance and salinity effects on plants: A review. Ecotoxicology and Environmental Safety. 60(3), 324-349.

Pesci, P. 1987. ABA-induced proline accumulation in barley leaf segments: dependence on protein synthesis. Physiol. Plant., 71(3), 287-291.

Piper, C.S. 1950 . Soil and plant analysis, ( $1^{\text {st }} \mathrm{Ed}$.). pp. 30-59, Inter. Science Publishers Inc., New York, USA. 
Effect of Saline Water Irrigation and Foliar Spraying of Salicylic Acid on Growth, Flowering and Chemical Composition of Pot Marigold (Calendula officinalis L.) Plant

Popova, L., Pancheva, T. and Uzunova, A. 1997. Salicylic acid: Properties, biosynthesis and physiological role. Bulg. J. Plant Physiol., 23, 85-93.

Raskin, I. 1992a. Role of salicylic acid in plants. Annual Review of Plant Physiology \& Plant Molecular Biology, 43, 439-463.

Raskin, I. 1992b. Salicylate, a new plant hormone. Plant Physiol., 99, 799-803.

Rivas-SanVicente, M. and Plasencia, J. 2011. Salicylic acid beyond defence: its role in plant growth and development. J. Exp. Bot., 62(10), 3321-3338.

Salachna, P., Piechocki, R., Zawadzińska, A. and Wośkowiak, A. 2015. Response of speckled spur-flower to salinity stress and salicylic acid treatment. J. Eco. Eng., 16(5), 68-75.

Sánchez-Blanco, M.J., Rodríguez, P., Morales, M.A. and Torrecillas, A. 2003. Contrasting physiological responses of dwarf sea-lavender and marguerite to simulated sea aerosol deposition. J. of Environment Quality. 32(6), 22382244.

Senaratna, T., Touchell, D., Bunn, E. and Dixon, K. 2000. Acetyl salicylic acid (Aspirin) and salicylic acid induce multiple stress tolerance in bean and tomato plants. Plant Growth Regulation, 30(2), 157-161.

Shakirova, F.M., Sakhabutdinova, A.R., Bezrukova, M.V., Fatkhutdinova, R.A. and Fatkhutdinova, D.R. 2003. Changes in the hormonal status of wheat seedlings induced by salicylic acid and salinity. Plant Science, 164(3), 317-322.
Syeed, S. 2008. Physiological basis for salicylic acid-mediated salinity tolerance of mungbean (Vicia radiata) and mustard (Brassica juncea).51 p., PhD thesis, Aligarh Muslim University, Aligarh, India.

Syeed, S. and Khan, N.A. 2010. Physiological aspects of salicylic acid-mediated salinity tolerance in plants. Plant stress. 4(1), 39-46.

Szepesi, A., Csiszár, J., Bajkán, S., Gémes, K., Horváth, F., Erdei, L., Deér, A.K., Simon, M.L. and Tari, I. 2005. Role of salicylic acid pre-treatment on the acclimation of tomato plants to salt- and osmotic stress. Acta Biologica Szegediensis, 49, 123-125.

Waller, R.A. and Duncan, D.B. 1969. A Bayes rule for the symmetric multiple comparison problem. J. of the American Statistical Association, 64, 1484-1503.

Williams, M., Senaratna, T., Dixon, K. and Sivasithamparam, K. 2003. Benzoic acid induces tolerance to biotic stress caused by Phytophthora cinnamomi in Banksia attenuata. Plant Growth Regul., 41(1), 89-91.

Wintermans, J.F. and De Mots, A. 1965. Spectrophotometric characteristics of Chlorophylls a and $b$ and their Phaeophytins in ethanol. Biochim. Biophys. Acta, 109(2), 448-453.

Yildirim, E., Turan, M. and Guvenc, I. 2008. Effect of foliar salicylic acid applications on growth, chlorophyll and mineral content of cucumber (Cucumis sativus L.) grown under salt stress. J. of Plant Nutrition, 31, 593-612. 